\title{
Proper Orthogonal Decomposition for Flow Calculations and Optimal Control in a Horizontal CVD Reactor *
}

\author{
Hung V. Ly ${ }^{1}$ and Hien T. Tran ${ }^{2}$ \\ Center for Research in Scientific Computation-Box 8205 \\ North Carolina State University \\ Raleigh, NC 27695-8205 \\ USA
}

March 23, 1998

\begin{abstract}
Proper orthogonal decomposition (which is also known as the Karhunen Loève decomposition) is a reduction method that is used to obtain low dimensional dynamic models of distributed parameter systems. Roughly speaking, proper orthogonal decomposition (POD) is an optimal technique of finding a basis which spans an ensemble of data, collected from an experiment or a numerical simulation of a dynamical system, in the sense that when these basis functions are used in a Galerkin procedure will yield a finite dimensional system with the smallest possible degrees of freedom. Thus the technique is well suited to treat optimal control and parameter estimation of distributed parameter systems. In this paper, the method is applied to analyze the complex flow phenomenon in a horizontal chemical vapor deposition (CVD) reactor. In particular, we show that POD can be used to efficiently approximate solutions to the compressible viscous flows coupled with the energy and the species equations. In addition, we also examined the feasibility and efficiency of POD method in the optimal control of the source vapors to obtain the most uniform deposition profile at the maximum growth rate. Finally, issues concerning the implementation of the method and numerical calculations are discussed.
\end{abstract}

AMS Subject Classification: $76 N 10,65 K 10,49 J 208335 \mathrm{C} 10$

*This research was supported in part by the DOD-MURI grant F49620-95-1-0447 through the Center for Intelligent Design and Manufacturing in Electronics and Materials.

${ }^{1}$ Email: hvly@eos.ncsu.edu.

${ }^{2}$ Email: tran@control.math.ncsu.edu. 


\section{Introduction}

Chemical vapor deposition (CVD) processes use a chemical reaction in the gas phase above the surface of the film to deposit desired materials onto a susceptor. CVD is a key element in a wide variety of industrial applications, ranging from the fabrication of microelectronic circuits, solar cells, and optical devices to the deposition of wear resistant coatings onto high performance machine tools. In a typical CVD reactor, a mixture of reactants and carrier gas is forced to flow across a heated susceptor. The temperature field from the heated susceptor induces gas phase reactions to produce activated species which then diffuse to the surface reaction layer and decompose to produce a thin film.

The deposited films, whose thicknesses range from a few nanometers to a few microns, must be produced with controllable properties such as purity, composition, thickness, microstructure, and surface morphology (see e.g., Jensen et al. (1991)). The tolerance limits on the properties of the films vary with the application. Currently, however, the required properties of the majority of industrially important thin films that are produced by CVD have become increasingly difficult to achieve. For example, some most important applications which pertain to infrared laser sources require micron thick films. This mandates epitaxial deposition at rates higher than those which can be achieved at low densities. The associated density gradients (due to large temperature gradients between the inlet and the susceptor) in a gravitational field will induce natural convection flows. Convection, in turn, influences the growth processes in two different ways, only one of which is beneficial. Convection increases mixing and the overall transport and, thus, the growth rate, which is desirable. On the other hand, it can also affect the morphology of the solid adversely. The latter phenomenon is due, in part, to the increased residential time resulting from the slow diffusional exchange of the reactant species between the main flow stream and laminar recirculation cells. Thus, for both the design of CVD reactors (including determining optimal operative conditions such as input flow rates) and the improvement of device properties, a qualitative and/or quantitative understanding of the transport processes in CVD reactors is of great importance.

In the past two decades steady progress has been made in the modeling of the transport processes in CVD reactors. For example, Moffat and Jensen (1986), (1988) used a fully parabolic flow approximation in axial direction for a three-dimensional (3D) horizontal reactor. Gokoglu et al. (1989) studied the deposition of $S i$ with a more detailed 3D flow treatment in a similar reactor geometry. In Ouazzani et al. (1988), (1990), various 2D and 3D models of a horizontal chemical vapor deposition reactor were investigated and the results were compared with experimental data. Studies also have been carried out to investigate crystal growth under reduced gravity conditions (see e.g., Ouazzani et al. (1988) and Scroggs et al. (1995)). One potential advantage of such conditions is that, under low gravity environment, buoyancy-driven convection is reduced (Ostrach (1982)). For an in-depth review of the work in these areas see the articles by Jensen (1989), Fotiadis (1990), and Jensen et al. (1991). Because of the complexity in CVD reactor models (represented by systems of nonlinear 
partial differential equations), the majority of the models are solved numerically by either finite-difference (Coltrin et al. (1984)), finite-volume (Ouazzani et al. (1990)), or finite-element methods (Jensen et al. (1991)). On the other hand, analytical investigations have used similarity transformations (Pollard and Newman (1980)) or separation of variable techniques (Fujii et al. (1972)). These approaches are restricted to one-dimensional, linear, and constant coefficient equations. Consequently, these models neglect some of the more important nonlinear effects in CVD processes such as buoyancy, temperature dependent flow parameters, and nonlinear coupling between the thermal, flow, and species fields. An asymptotic analysis of a CVD system which included temperature dependent coefficients, nonlinear coupling of transport processes and Soret diffusion effects was given by Young et al. (1992).

In addition to the above parameter studies (effects of operating conditions, reactor geometry, and heat transfer characteristics on flow patterns and growth rate uniformity), a more rigorous approach to the optimal design and control has also been investigated. In Ito, et al. (1994), a shape optimization problem with respect to the geometry of the reactor and a boundary temperature control problem were formulated. The material and shape derivatives of solutions to the Boussinesq approximation were derived. Optimality conditions and a numerical optimization method based on the augmented Lagrangian method were developed for boundary control of Boussinesq flow. Numerical calculations in Ito et al. (1995) indicated the effectiveness of temperature control through a portion of the boundary for improving the vertical transport of flow in the cavity.

Numerical simulation has been shown to be an effective tool for the understanding and improvement of CVD processes. Of particular interest to the present investigation is the development of a framework to extend these simulation capabilities into the realm of optimal design and optimal control of CVD reactors. For the optimal control of CVD processes, we begin by defining a set of process parameters which control the system (e.g., flow rates, species concentration), and cost and constraint functions which quantify the desirable quantities of the response (e.g., growth rate and uniform fluxes of reactants at the susceptor). We then perform the simulation of the system process, and subsequently evaluate the cost and the constraint functions. These data are then supplied to some numerical optimization routine which modifies the process parameters to reduce the cost functional value and to satisfy the constraints (Banks, et al. (1997)). The complex fluid dynamics in CVD processes are described by a system of nonlinear partial differential equations representing the continuity, momentum, energy, species and equation of state. Therefore, numerical simulations of such systems using finite element, finite volume, finite difference, or spectral methods will lead, in general, to a very large system of ordinary differential equations rendering it inapplicable in real time estimation and control. One approach to overcome these difficulties is to perform model-predictive control of these distributed parameter systems. Although this method has the merit of small degrees of freedom, they do not represent the physical model, but rather an empirical model that is based on input and output of a given system 
and may become unstable as the operating condition of the system changes. For an efficient real-time control of CVD reactors where continuous computations must be performed, not only the degrees of freedom of the dynamic model must be small but the dynamic model must also be robust. In this work, we demonstrate the feasibility and efficiency of the proper orthogonal decomposition technique (or the Karhunen-Loève procedure) in flow calculations and the optimal control of CVD processes. The proper orthogonal decomposition (POD) is a reduction method that has been shown to be an effective tool for the analysis of complex systems such as turbulence flows, shear flows, pattern recognition, and weather prediction (see e.g., Berkooz, et al. (1993) and the references therein). In general, the discretization of nonlinear partial differential equations using finite element, finite volume, or finite different methods involves basis functions that have little to do with the differential equation. For example, piecewise polynomials are used in the finite element method, grid functions are used in the finite difference method, and Legendre or Chebyshev polynomials are used in some spectral methods. POD, on the other hand, uses basis functions that span a data set, collected from an experiment or numerical simulation of a dynamical system, in a certain "optimal" fashion. Because POD basis elements are optimal in the sense that they are the extractions of characteristic features of the data set, only a small number of POD basis functions are needed to describe the solution.

The organization of this paper is as follow. We describe in $\S 2$ a horizontal CVD reactor and its mathematical description. The POD technique and its mathematical properties are presented in $\S 3$. In $\S 4$ we discuss issues concerning the implementation and numerical calculations using POD in the context of simulating the flow dynamics in the CVD process. Finally, in $\$ 5$ the method is applied to solve an optimal control problem to achieve film uniformity and maximum growth rate.

\section{Formulation of the Problem}

The particular geometry of the organo-metallic chemical vapor deposition (OMCVD) reactor under consideration here features horizontal flow of the process gases and source vapor/carrier gas mixtures into an expansion section leading into a rectangular channel that contains the substrate (see Figure 1). The substrate wafer is mounted on a rotating induction heated $\mathrm{SiC}$ coated graphite susceptor. The exhaust gases are vented through a vertical exhaust tube. Loading and unloading of substrate wafers is accomplished through a load-lock chamber beneath the radio frequency (rf) section of the reactor that can be evacuated by a turbomolecular pump. After purging with ultra-pure nitrogen, sample transfer can be executed using a magnetic transfer rod. Gas is purging through the gap between the susceptor and the reactor's base to avoid flow of gas mixtures to the mechanical workings behind the susceptor. The quartz glass reactor is connected at the inlet to a source vapor/process gas flow control and switching panel that directs individual streams of source vapor saturated carrier gas either to a vent line or to the reactor. Thus, pulsed operation separating plugs of 


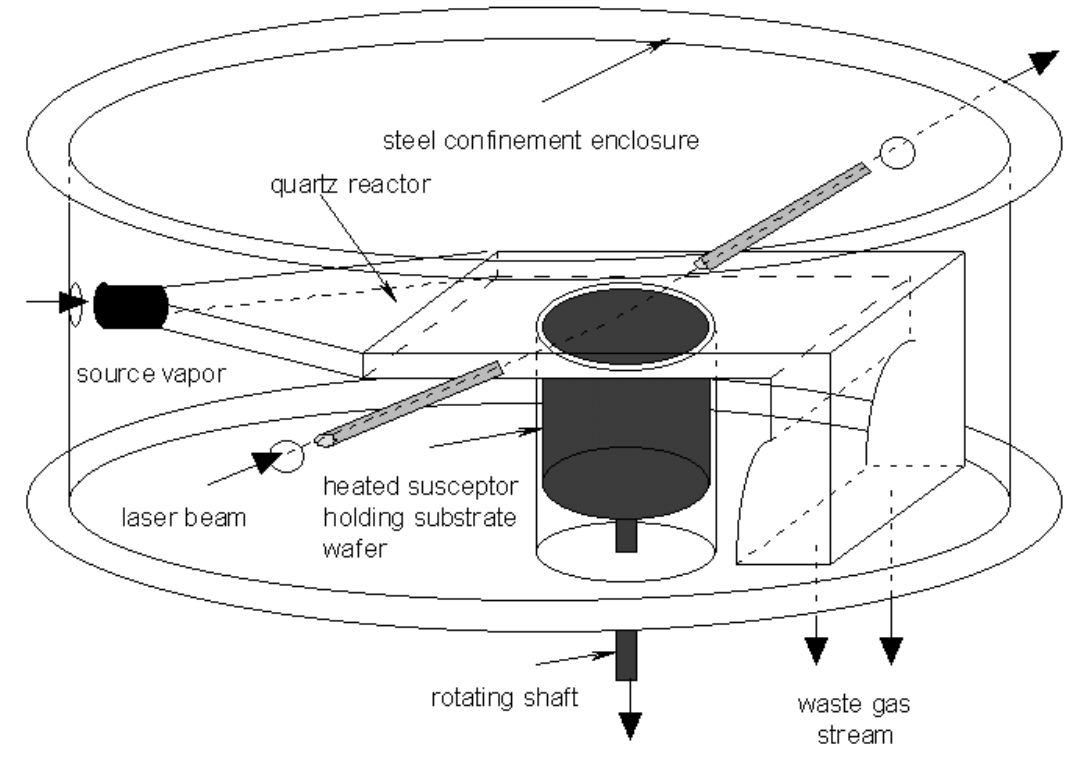

to load-lock chamber

Figure 1: Schematic representation of a horizontal, quartz reactor in a steel confinement shell

source vapor saturated carrier gas by plugs of high purity carrier gas, flow rate modulated flow or continuous flow can be implemented for all source vapors without change in reactor pressure or total flow. Two optical windows at the Brewster angle of the substrate are attached to the sides of the reactor. They allow for the real-time process monitoring utilizing p-polarized reflectance spectroscopy (PRS) (see e.g., Bachmann, et al (1998) and the references therein). This OMCVD reactor has been built in the laboratory of Prof. Klaus Bachmann at North Carolina State University and is now undergoing initial testing.

To demonstrate that the POD method can be implemented to approximate transport processes efficiently in the CVD reactor, we will restrict our study to a two-dimensional horizontal reactor as shown in Figure 2 (this can be thought of as a vertical "slice" of the actual 3-D reactor tube). Our study involving the full 3-dimensional geometry will be presented in subsequent papers. We will consider the deposition of $\mathrm{GaN}$ using pulsed trimethyl-gallium (TMGa) and ammonia $\left(\mathrm{NH}_{3}\right)$ as source vapors and nitrogen as carrier gas (see Figure 3). The function $F(t)$ in Figure 3 represents the pulses of reactive gases entering the reactor. In particular, at first only carrier gas flows through the reactor. After the flow reaches steady state, a pulse of reactant (e.g., TMGa) diluted with carrier gas enters the reactor. After the pulse, the reactor is then flushed with carrier gas. This process is then repeated for another reactant. Furthermore, the following assumptions are made for the mathematical formulation of this 
process.

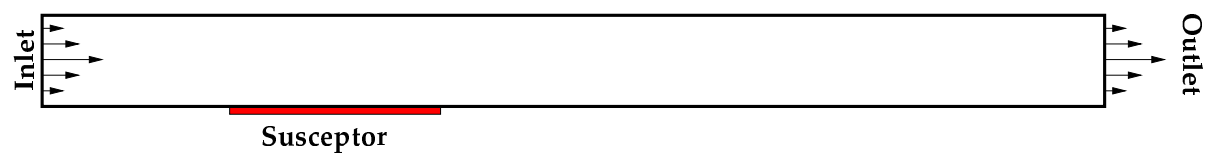

Figure 2: Two-dimensional horizontal reactor

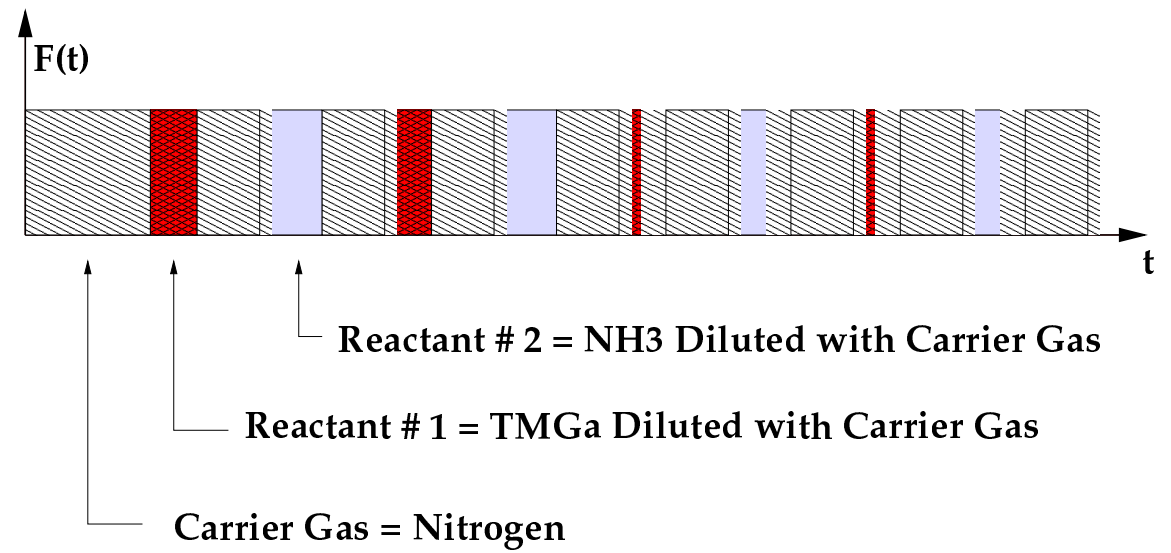

Figure 3: Chemicals introduced into the reactor at the inlet

(i) Carrier gas flows through the reactor at all time.

(ii) All thermo-physical properties, conductivity, viscosity, mass diffusivity, and volume expansion are temperature dependent.

(iii) Only a trace amount of reactants mixed with carrier gas is allowed to enter the reactor at each pulse so that the steady state gas flow condition and all temperature dependent parameters in (ii) remain unchanged.

(iv) No chemical reaction takes place in the gas phase.

(v) Reactions taking place on the heated substrate are very fast and equilibrium is attained quickly. Thus, the rate of GaN deposition is limited by mass transport.

(vi) The wall of the reactor is water cooled.

Under the above assumptions, CVD processes can be classified as a quasitransient flow (steady-state flow with transient species) and be described by the following governing partial differential equations written in conservation form (see e.g., Oran and Boris [1987] and Oswatitsch [1956]). 
Mass conservation equation:

$$
\nabla \cdot(\rho \overrightarrow{\mathbf{u}})=0
$$

where $\rho$ is the density of the carrier gas and $\overrightarrow{\mathbf{u}}=\left(u_{1}, u_{2}\right)$ is the velocity vector.

Momentum conservation equation:

$$
\rho \overrightarrow{\mathbf{u}} \cdot \nabla \overrightarrow{\mathbf{u}}=-\nabla P+\nabla \cdot \sigma+\left(\rho-\rho_{0}\right) g \vec{j}
$$

where the viscous stress tensor $\sigma$ has the form

$$
\sigma=\left[\mu_{T}\left(\nabla \overrightarrow{\mathbf{u}}+(\nabla \overrightarrow{\mathbf{u}})^{\mathcal{T}}\right)\right]+\left(\kappa-\frac{2}{3} \mu_{T}\right)(\nabla \cdot \overrightarrow{\mathbf{u}}) \cdot I .
$$

Here we treat the gas mixture as a Newtonian fluid and $P$ is the pressure, $\mu_{T}$ is the carrier gas viscosity, $\kappa$ is the bulk viscosity which can be neglected for dense gases or liquids, $g$ is the gravitational force, and $\rho_{0}$ is the reference density. The $\left(\rho-\rho_{0}\right) g \vec{j}$ term accounts for the natural convection effect caused by the gravitational force.

Energy conservation equation:

$$
c_{p} \rho \overrightarrow{\mathbf{u}} \cdot \nabla T=\nabla \cdot\left(\lambda_{T} \nabla T\right)
$$

where $T$ is the temperature, and $c_{p}$ is the heat capacity and $\lambda_{T}$ is thermal conductivity of the carrier gas.

Mass conservation of species:

$$
\frac{\partial c}{\partial t}+\overrightarrow{\mathbf{u}} \cdot \nabla c=\frac{1}{\rho} \nabla \cdot\left(\rho D_{T} \nabla c\right),
$$

where $c$ is the mass fraction of TMGa and $D_{T}$ is the diffusion coefficient of TMGa in the carrier gas. Here, without loss of generality, only the transport of TMGa is modeled.

Expansion of the gas as it approaches the heated susceptor plays a major role in the flow behavior and it is accounted for by using the following Boussinesq approximation for the density as a function of the temperature:

$$
\rho=\rho_{0}\left[1-\beta_{T}\left(T-T_{0}\right)\right]
$$

where $\beta_{T}$ is the volume expansion and $T_{0}$ is a reference temperature.

The boundary conditions for the above set of equations (2.2)-(2.5) are summarized in Figure 4, where the parabolic profile for the velocity field at the inlet is described by

$$
u_{1}(t)=\frac{U_{\max }}{4 h^{2}} y(H-y) \quad \text { and } \quad u_{2}(t)=0
$$

with $U_{\max }=0.25 \mathrm{~m} / \mathrm{s}$ and $H=0.535$ inch is the height of the reactor. The initial condition for the species equation is zero throughout the domain of the rectangular reactor. 


\begin{tabular}{|c|c|c|c|}
\hline $\begin{array}{c}\text { Boundary } \\
\text { conditions }\end{array}$ & Velocity & Temperature & Species \\
\hline Inlet & Parabolic profile & $298 \mathrm{~K}$ & $\mathrm{~F}(\mathrm{t})$ \\
\hline Walls & 0 & $298 \mathrm{~K}$ & $\mathrm{dc} / \mathrm{dn}=0$ \\
\hline Susceptor & 0 & $1200 \mathrm{~K}$ & 0 \\
\hline Outlet & $\mathrm{du} \mathbf{i}_{\mathbf{i}} / \mathrm{dn}=\mathbf{0 , i = 1 , 2}$ & $\mathrm{dT} / \mathrm{dn}=0$ & $\mathrm{dc} / \mathrm{dn}=0$ \\
\hline
\end{tabular}

Figure 4: Boundary Conditions for the 2D Horizontal Reactor

In this model, one proceeds first by solving for the steady state solutions of the flow and energy equations. These solutions are then substituted into equation (2.4) to solve for the time dependent species solution. Even though the flow and energy solution are decoupled from the mass transport analysis, the dynamical model is still an infinite dimensional system of equations. Standard techniques such as finite difference or finite element methods can be employed to reduce the infinite dimensional models to finite dimensional ones, but the resulting degrees of freedom are, in general, too large for practical considerations in estimation or control.

It will be demonstrated in the sequel that a Galerkin procedure employing basis functions which are computed from the proper orthogonal decomposition can efficiently reduce distributed parameter systems to low order finite dimensional dynamical models while maintaining high fidelity. Thus, this approach is particularly suitable to treat optimal control and parameter estimation problems of systems governed by partial differential equations.

\section{Proper Orthogonal Decomposition}

The proper orthogonal decomposition (POD) method has received much attention in recent years as a tool to analyze complex physical systems. In principle, the idea is to use a reliable solver to produce a priori a number of solutions to the physical model (called snapshots). The POD technique is then used to produce an "optimal" representation of these snapshots in an "average" sense. Both notions, "optimal" and "average", will be made clear in subsequent discussions. The power of the POD method lies in its mathematical properties which suggest that it is the preferred method to use in many applications.

Proper orthogonal decomposition was independently proposed by several 
scientists including Karhunen (1946), Loève (1945), Pougachev (1953), and Obukhov (1954) (for recent surveys in this area see the works of Lumley (1970) and Berkooz (1992)). Some mathematical theories of POD can be found in recent articles by Aubry, Lian and Titi (1993) and Graham and Kevrekidis (1996). The POD technique has been applied to numerous applications. One such important application was the attraction of spatial organized motions in fluid flows. Theodorsen (1952) and later Townsend (1956) observed and indicated that there are large-scale organized motions embedded in turbulent shear flows. Lumley (1967), Aubry, Holmes, Lumley and Stone (1988), Sirovich (1991), Berkooz, Holmes and Lumley (1993), Berkooz, Holmes, Lumley and Mattingly (1997) have adapted the POD technique to study turbulent flows. Other applications of POD include channel flows by Moin and Moser (1989), Ball, Sirovich and Keefe (1991), square-duct flows by Reichert, Hatay, Biringer and Husser (1994), and shear flows by Rajaee, Karlson and Sirovich (1993), Kirby, Boris and Sirovich (1990). Other scientists have also applied the POD technique to fluid related problems. For instance, it has been applied to the Burgers' equation by Chambers, Adrian, Moin, Stewart and Sung (1988), the Ginzburg-Landau equation and the Bénard convection by Sirovich (1989). Ly and Tran (1998) have used POD to simulate and solve an optimal control problem for Rayleigh-Bénard convection. Other interesting non-fluid applications of POD techniques are the characterization of human faces by Kirby and Sirovich (1990) and image recognition by Hilai and Rubinstein (1994).

As will be seen in the following section, one reason that POD is an attractive method is that it is a linear procedure. Its mathematical theory is based on the spectral theory of compact, self-adjoint operators. However, it should be noted that POD makes no assumption on the linearity of the problem to which it is applied and this is an extremely positive feature of this approach to model reduction.

\subsection{Mathematical Aspects of POD}

Let $\left\{\mathbf{U}_{i}(x): 1 \leq i \leq N ; x \in \Omega\right\}$ denote the set of $N$ observations (also called snapshots) of some physical processes over a domain $\Omega$. In the context of CVD process, these observations could be experimental measurements or numerical solutions of velocity fields, temperatures, species etc. taken at different physical parameters (Reynolds number, input flow rates etc.) or time steps. The POD technique is designed to extract from this set of observations a coherent structure, which has the largest mean square projection on the observations. In other words, we look for a function $\Phi$, or the so-called POD basis element, that most resembles $\left\{\mathbf{U}_{i}(x)\right\}_{i=1}^{N}$ in the sense that it maximizes

$$
\frac{1}{N} \sum_{i=1}^{N}\left|\left(\mathbf{U}_{i}, \Phi\right)\right|^{2},
$$

subject to

$$
(\Phi, \Phi)=\|\Phi\|^{2}=1
$$


where $(\cdot, \cdot)$ and $\|\cdot\|$ denote the usual $\mathbf{L}^{2}$ inner product and $\mathbf{L}^{2}$-norm over $\Omega$, respectively. We choose a special class of trial functions for $\Phi$ to be of the form:

$$
\Phi=\sum_{i=1}^{N} a_{i} \mathbf{U}_{i}
$$

where the coefficients $a_{i}$ are to be determined so that $\Phi$ given by the expression (3.2) provides a maximum for (3.1). To this end, let us define

$$
\mathbf{K}\left(x, x^{\prime}\right):=\frac{1}{N} \sum_{i=1}^{N} \mathbf{U}_{i}(x) \mathbf{U}_{i}\left(x^{\prime}\right) \quad \text { and } \quad \mathbf{R} \Phi:=\int_{\Omega} \mathbf{K}\left(x, x^{\prime}\right) \Phi\left(x^{\prime}\right) d x^{\prime},
$$

where $\mathbf{R}: \mathbf{L}^{2}(\Omega) \rightarrow \mathbf{L}^{2}(\Omega)$.

Then straightforward calculations reveal that

$$
\begin{aligned}
(\mathbf{R} \Phi, \Phi) & =\int_{\Omega} \mathbf{R} \Phi(x) \Phi(x) d x \\
& =\int_{\Omega} \int_{\Omega} \mathbf{K}\left(x, x^{\prime}\right) \Phi\left(x^{\prime}\right) d x^{\prime} \Phi(x) d x \\
& =\frac{1}{N} \sum_{i=1}^{N} \int_{\Omega} \int_{\Omega} \mathbf{U}_{i}(x) \mathbf{U}_{i}\left(x^{\prime}\right) \Phi\left(x^{\prime}\right) d x^{\prime} \Phi(x) d x \\
& =\frac{1}{N} \sum_{i=1}^{N}\left|\left(\mathbf{U}_{i}, \Phi\right)\right|^{2} .
\end{aligned}
$$

Furthermore, it follows that

$$
(\mathbf{R} \Phi, \Psi)=(\Phi, \mathbf{R} \Psi) \quad \text { for any } \quad \Phi, \Psi \in \mathbf{L}^{2} .
$$

Thus $\mathbf{R}$ is a nonnegative symmetric operator on $\mathbf{L}^{2}(\Omega)$. Consequently, the problem of maximizing the expression (3.1) amounts to finding the largest eigenvalue to the eigenvalue problem

$$
\mathbf{R} \Phi=\lambda \Phi \quad \text { subject to } \quad\|\Phi\|=1
$$

or

$$
\int_{\Omega} \mathbf{K}\left(x, x^{\prime}\right) \Phi\left(x^{\prime}\right) d x^{\prime}=\lambda \Phi \quad \text { with } \quad\|\Phi\|=1 .
$$

Substituting expression (3.2) and the definition of $\mathbf{K}$ into equation (3.4), we obtain

$$
\sum_{i=1}^{N}\left[\sum_{k=1}^{N}\left(\frac{1}{N} \int_{\Omega} \mathbf{U}_{i}\left(x^{\prime}\right) \mathbf{U}_{k}\left(x^{\prime}\right) d x^{\prime}\right) a_{k}\right] \mathbf{U}_{i}(x)=\sum_{i=1}^{N} \lambda a_{i} \mathbf{U}_{i}(x) .
$$

This can be rewritten as the eigenvalue problem

$$
\mathbf{C V}=\lambda \mathbf{V}
$$


where

$$
\mathbf{C}_{i k}=\frac{1}{N} \int_{\Omega} \mathbf{U}_{i}(x) \mathbf{U}_{k}(x) d x \quad \text { and } \quad \mathbf{V}=\left[\begin{array}{c}
a_{1} \\
a_{2} \\
\vdots \\
a_{N}
\end{array}\right]
$$

Since $\mathbf{C}$ is a nonnegative Hermitian matrix, it has a complete set of orthogonal eigenvectors

$$
\mathbf{V}^{1}=\left[\begin{array}{c}
a_{1}^{1} \\
a_{2}^{1} \\
\vdots \\
a_{N}^{1}
\end{array}\right], \mathbf{V}^{2}=\left[\begin{array}{c}
a_{1}^{2} \\
a_{2}^{2} \\
\vdots \\
a_{N}^{2}
\end{array}\right], \ldots, \mathbf{V}^{N}=\left[\begin{array}{c}
a_{1}^{N} \\
a_{2}^{N} \\
\vdots \\
a_{N}^{N}
\end{array}\right]
$$

with the corresponding eigenvalues $\lambda_{1} \geq \lambda_{2} \geq \cdots \geq \lambda_{N} \geq 0$. Thus, the solution to the optimization problem for (3.1) is given by

$$
\Phi_{1}=\sum_{i=1}^{N} a_{i}^{1} \mathbf{U}_{i}
$$

where $a_{i}^{1}$ are the elements of the eigenvector $\mathbf{V}^{1}$ corresponding to the largest eigenvalue $\lambda_{1}$. The remaining POD basis elements, $\Phi_{i}, i=2, \ldots, N$, are obtained by using the elements of other eigenvectors, $\mathbf{V}^{i}, i=2, \ldots, N$. Moreover using the orthogonality of $\left\{\mathbf{V}^{k}: 1 \leq k \leq N\right\}$ and the imposed condition:

$$
\mathbf{V}^{k} \cdot \mathbf{V}^{k^{\prime}}=\sum_{i=1}^{N} a_{i}^{k} a_{i}^{k^{\prime}}= \begin{cases}\frac{1}{N \lambda_{k^{\prime}}} & k=k^{\prime} \\ 0 & k \neq k^{\prime}\end{cases}
$$

we obtain

$$
\begin{aligned}
\left(\Phi_{k}, \Phi_{k^{\prime}}\right) & =\int_{\Omega} \Phi_{k}(x) \Phi_{k^{\prime}}(x) d x \\
& =\int_{\Omega} \sum_{i=1}^{N} a_{i}^{k} \mathbf{U}_{i}(x) \sum_{j=1}^{N} a_{j}^{k^{\prime}} \mathbf{U}_{j}(x) d x \\
& =\sum_{i=1}^{N} a_{i}^{k} N \sum_{j=1}^{N}\left(\frac{1}{N} \int_{\Omega} \mathbf{U}_{i}(x) \mathbf{U}_{j}(x) d x\right) a_{j}^{k^{\prime}} \\
& =\sum_{i=1}^{N} a_{i}^{k} N \sum_{j=1}^{N} \mathbf{C}_{i j} a_{j}^{k^{\prime}} \\
& =N \mathbf{V}^{k} \cdot \mathbf{C} \mathbf{V}^{k^{\prime}} \\
& =N \mathbf{V}^{k} \cdot \lambda_{k^{\prime}} \mathbf{V}^{k^{\prime}} \\
& =N \lambda_{k^{\prime}} \mathbf{V}^{k} \cdot \mathbf{V}^{k^{\prime}} \\
& = \begin{cases}1 & k=k^{\prime} \\
0 & k \neq k^{\prime} .\end{cases}
\end{aligned}
$$


Thus, the POD basis $\left\{\Phi_{1}, \Phi_{2}, \ldots, \Phi_{N}\right\}$ forms an orthonormal set.

Remarks. An alternative approach for finding the solution to maximization of (3.1) is by using the so-called Rayleigh-Ritz method for finding eigenvalues (see Kanwal (1997) p. 176). Since

$$
(\mathbf{R} \Phi, \Phi)=\left(\mathbf{R}\left[\sum_{i=1}^{N} a_{i} \mathbf{U}_{i}\right], \sum_{k=1}^{N} a_{k} \mathbf{U}_{k}\right)=\sum_{i=1}^{N} \sum_{k=1}^{N} \mathbf{R}_{i k} a_{i} a_{k},
$$

and

$$
\|\Phi\|^{2}=(\Phi, \Phi)=\sum_{i=1}^{N} \sum_{k=1}^{N} N \mathbf{C}_{i k} a_{i} a_{k},
$$

where $\mathbf{R}_{i k}=\left(\mathbf{R} \mathbf{U}_{i}, \mathbf{U}_{k}\right)=\left(\mathbf{U}_{i}, \mathbf{R} \mathbf{U}_{k}\right)$ and $\mathbf{C}_{i k}=\frac{1}{N}\left(\mathbf{U}_{i}, \mathbf{U}_{k}\right)=\frac{1}{N}\left(\mathbf{U}_{k}, \mathbf{U}_{i}\right)$ as above in (3.5), the problem of maximizing (3.1) can be transformed into an extremal problem in multi-variable calculus with $\left\{a_{1}, a_{2}, \ldots, a_{N}\right\}$ as variables. Recalling the method of Lagrange multipliers, we define

$$
\mathbf{G}\left(a_{1}, \ldots, a_{N}\right):=\sum_{i=1}^{N} \sum_{k=1}^{N} \mathbf{R}_{i k} a_{i} a_{k}-\lambda N \mathbf{C}_{i k} a_{i} a_{k},
$$

where $\lambda$ is the Lagrange multiplier. Equating $\partial \mathbf{G} / \partial a_{i}$ to 0 (a necessary condition for optimality) we find

$$
\sum_{k=1}^{N}\left(\mathbf{R}_{i k}-\lambda N \mathbf{C}_{i k}\right) a_{k}=0 \quad \text { for } \quad i=1, \ldots, N .
$$

Equation (3.6) has a nontrivial solution if and only if the determinant of $(\mathbf{R}-\lambda N \mathbf{C})=0$. This determinant, when expanded, yields an $N$ th degree polynomial in $\lambda$ which can be shown to have $N$ nonnegative real roots, but not necessarily distinct (these can be ordered in descending order as $\lambda_{1} \geq \lambda_{2} \geq \cdots \geq \lambda_{N}$ ) (Kanwal (1997)). Moreover by multiplying equation (3.6) by $a_{i}$ and summing over $i=1, \ldots, N$, we obtain $\lambda=(\mathbf{R} \Phi, \Phi)$. It can be shown that solution pair $\left(\lambda_{i}, V^{i}\right)$ where $\mathbf{V}^{i}=\left(a_{1}^{i}, \ldots, a_{N}^{i}\right)$, is the eigenvalue and eigenvector of the matrix $\mathbf{C}$. One then uses the definition of $\Phi$ in (3.2) to obtain $\left\{\Phi_{1}, \Phi_{2}, \ldots, \Phi_{N}\right\}$ and uses the fact that the matrix $\mathbf{C}=\left[\mathbf{C}_{i j}\right]$ is Hermitian to justify the orthonormality of $\left\{\Phi_{k}\right\}$ 's as in previous argument.

\subsection{Optimality of the POD Basis}

Suppose that we have a signal $v(x, t)$ with $v \in L^{2}(\Omega,[0, T])$ and an approximation of $v^{N}$ of $v$ with respect to an arbitrary orthonormal basis $\psi_{i}(x), i=$ $1,2, \ldots, N$ :

$$
v^{N}(x, t)=\sum_{i=1}^{N} a_{i}(t) \psi_{i}(x)
$$


If the $\psi_{i}(x)$ have been nondimensionalized, then the coefficients $a_{i}$ carry the dimension of the quantity $v^{N}$. If $v^{N}(x, t)$ denotes the velocity and $\langle\cdot>$ denotes the time average operator, then the average kinetic energy per unit mass is given by

$$
<\int_{\Omega} v^{N}(x, t) v^{N^{*}}(x, t) d x>=<\sum_{i=1}^{N} a_{i}(t) a_{i}^{*}(t)>.
$$

Consequently, the expression $\left\langle a_{i} a_{i}^{*}>\right.$ represents the average kinetic energy in the $\mathrm{i}^{\text {th }}$-mode. The following lemma establishes the notion of optimality of the POD method.

Lemma 3.1 Let $\left\{\Phi_{1}, \Phi_{2}, \ldots, \Phi_{N}\right\}$ denote the orthonormal set of POD basis elements and $\left(\lambda_{1}, \lambda_{2}, \ldots, \lambda_{N}\right)$ denote the corresponding set of eigenvalues. If

$$
v^{N}(x, t)=\sum_{i=1}^{N} b_{i}(t) \Phi_{i}(x)
$$

denotes the approximation to $v$ with respect to this basis, then the following hold:

(a) $\left\langle b_{i}(t) b_{j}^{*}(t)\right\rangle=\delta_{i j} \lambda_{i}$ (that is, the POD coefficients are uncorrelated).

(b) For every $N$,

$$
\sum_{i=1}^{N}<b_{i}(t) b_{i}^{*}(t)>=\sum_{i=1}^{N} \lambda_{i} \geq \sum_{i=1}^{N}<a_{i}(t) a_{i}^{*}(t)>.
$$

The proof of this lemma is straight forward from the optimality of the eigenvalues and can be found in Berkooz, Holmes, and Lumley (1993). This lemma establishes that among all linear combinations, the POD is the most efficient, in the sense that, for a given number of modes, $N$, the projection on the subspace used for approximation will contain the most kinetic energy possible in an average sense.

\subsection{Model Reduction Features of POD Approximations}

To this point we have not discussed any model reduction features associated with using POD basis elements in approximation schemes. In the construction described above, the number $N$ may be large, $100-1000$ or even more, depending on the complexity of the dynamics represented in the "snapshots" $\mathbf{U}_{i}$. In general, one should take $N$ sufficiently large so that the snapshots $\mathbf{U}_{i}$ contain all salient features of the dynamics being investigated. Thus, the POD basis functions $\Phi_{i}$, used with the original dynamics in a nonlinear Galerkin procedure, offers the possibilities of achieving a high fidelity model, albeit with perhaps a large dimension $N$.

To achieve model reduction, one chooses $M \ll N$ and carries out a nonlinear Galerkin procedure with the set of elements $\left\{\Phi_{1}, \Phi_{2}, \ldots, \Phi_{M}\right\}$. The crucial 
question is how to choose $M$. As we indicated in the previous section, $\sum_{i=1}^{M} \lambda_{i}$ represents the average kinetic energy contained in the first $M$ modes and hence to capture most of the energy of the system contained in the $N$ POD elements, it suffices to choose $M$ so that $\sum_{i=1}^{M} \lambda_{i} \approx \sum_{i=1}^{N} \lambda_{i}$. Indeed, the ratio $\sum_{i=1}^{M} \lambda_{i} / \sum_{i=1}^{N} \lambda_{i}$ yields the percentage of the total kinetic energy in the $N$ POD elements that is contained in the first $M$ POD elements. Since the associated POD eigenvalues are ordered $\lambda_{1} \geq \lambda_{2} \geq \cdots \geq \lambda_{N}$, one can reasonably expect to achieve a high percentage of the total kinetic energy in a reduced model of order $M$ with $M$ sufficiently smaller than $N$. For the CVD examples detailed below, the POD system was constructed for $N=200$ and a reduced order model with $M=10$ yielded a ratio of .999 , resulting in a truly significant computational savings over the finite element model (2,400 quadrilateral elements) of dimension 14,801 used to generate the 200 snapshots.

\section{Application of POD for the Simulation of CVD Processes}

We return to the CVD example of $\S 2$ to apply the POD techniques. Under assumption (iii) of $\S 2$, that is only a trace amount of TMGa mixed with carrier gas is used, the steady state flow and energy solutions can be decoupled from the mass transport equation. Therefore, we first solve equations (2.1)-(2.3) and (2.5) for the steady state solutions of velocity field, temperature, density and pressure. These solutions, depicted graphically in Figure 5, are computed using a commercial fluid dynamics package called FIDAP version 7.6 which employs the finite element method. In our simulations we used 2,400 (9-nodal quadratic) quadrilateral elements. Here and in all subsequent plots, higher numerical values are represented by darker shaded region.

Once the flow has reached steady state condition in the reactor, we introduce TMGa into the reactor from the inlet. As mentioned earlier, the term $F(t)$ used in the boundary condition for the species at the inlet, $\left.c(\vec{x}, t)\right|_{\text {inlet }}=F(t)$, describes the incoming pulses of TMGa. Using the steady state velocity and density solutions (for the terms $\overrightarrow{\mathbf{u}}$ and $\rho$ ) and the steady state temperature solution (to compute temperature dependent mass diffusivity $D_{T}$ ) in equation (2.4), we simulated the species equation for zero initial condition and various boundary conditions $F(t)$ using FIDAP. Figure 6 displays TMGa profiles at different time steps from 0.05 to 0.75 seconds corresponding to the boundary condition $\left.c(\overrightarrow{\mathbf{x}}, t)\right|_{\text {inlet }}=F(t)=1$, for all $t>0$. We note that, depending on the duration of TMGa introduced at the inlet, different TMGa profiles will result in the reactor. In particular, for the following boundary condition (see also Figure 8):

$$
\left.c(\overrightarrow{\mathbf{x}}, t)\right|_{\text {inlet }}= \begin{cases}F(t)=1 & 0<t \leq \mathrm{T}_{\text {in }} \\ 0 & \mathrm{~T}_{\text {in }}<t \leq \mathrm{T}_{\text {in }}+\mathrm{T}_{\text {out }} .\end{cases}
$$

Figure 7 depicts two different reactant profiles at different time steps from 0.05 second to $\mathrm{T}_{\text {out }}=0.4$ second corresponding to $\mathrm{T}_{\mathrm{in}}=0.3$ second and $\mathrm{T}_{\mathrm{in}}=$ 0.75 second. In the sequel, we will demonstrate that the proper orthogonal 

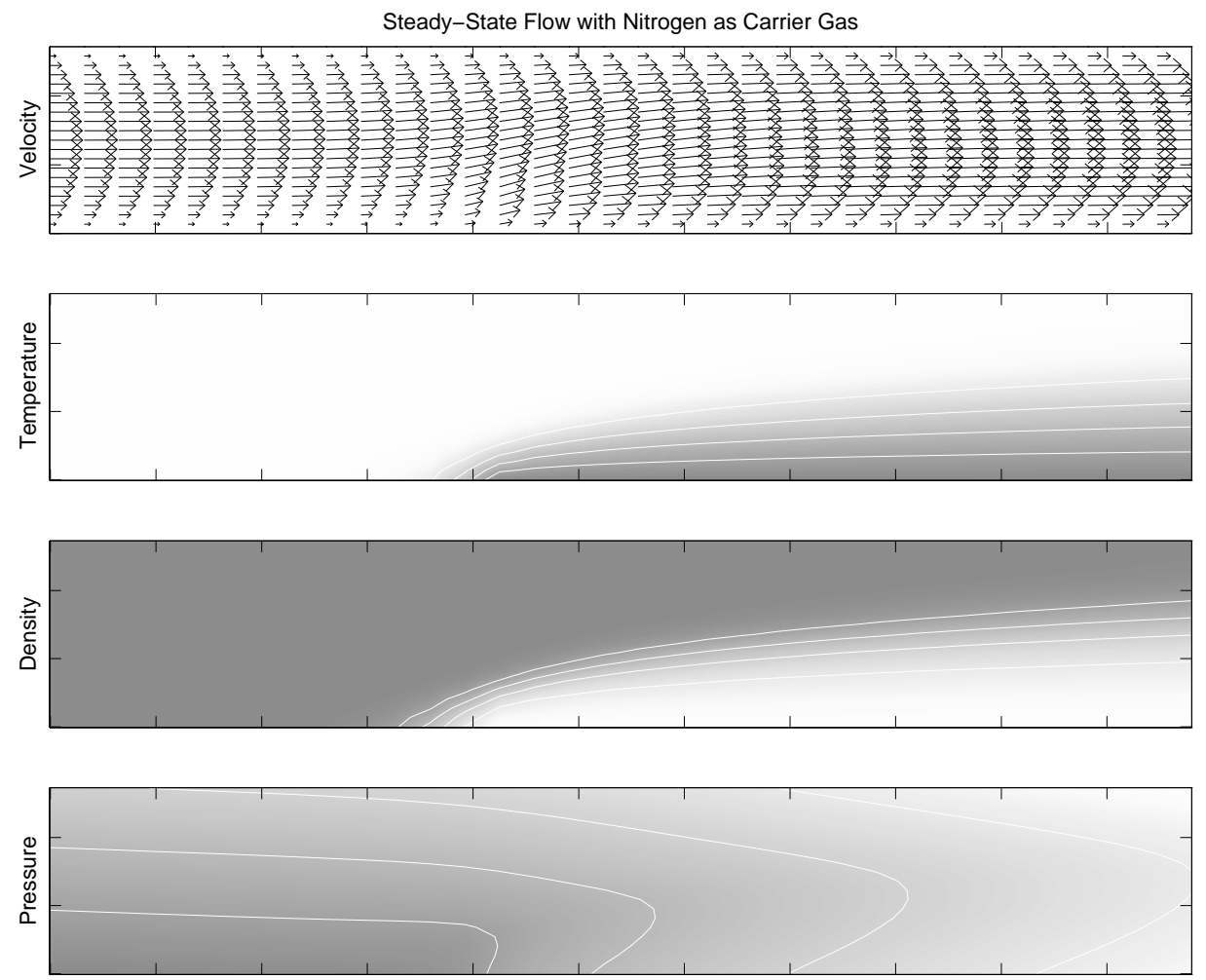

Figure 5: Steady state velocity field, temperature, density and pressure

decomposition method implemented in a Galerkin procedure can be used to simulate both accurately and efficiently the complicated species dynamics for different boundary conditions (i.e., different $\mathrm{T}_{\text {in }}$ values).

\subsection{Construction of POD Basis Vectors}

Consider the following semi-discrete nonlinear equation of the form

$$
\frac{d w}{d t}=\mathcal{G}(t, w(t)), \quad \text { for } t \in R, \quad w \in X, \quad \mathcal{G}: R \times X \rightarrow X,
$$

where $X$ is a finite-dimensional space. If finite element procedures were used to obtain this semi-discrete problem, then the choice for $X$ would be defined by $\operatorname{span}\left\{\varphi_{1}, \varphi_{2}, \ldots, \varphi_{N}\right\}$ where, for example, $\varphi_{i}$ are piecewise polynomial functions (e.g., the so-called hat functions, or spline functions). In the POD technique, however, we will make a different choice for the approximating space. Let $X^{\mathrm{POD}}$ denote the POD space such that $X^{\mathrm{POD}} \subset X$. The procedure for computing $X^{\mathrm{POD}}$ consists of the following steps. 


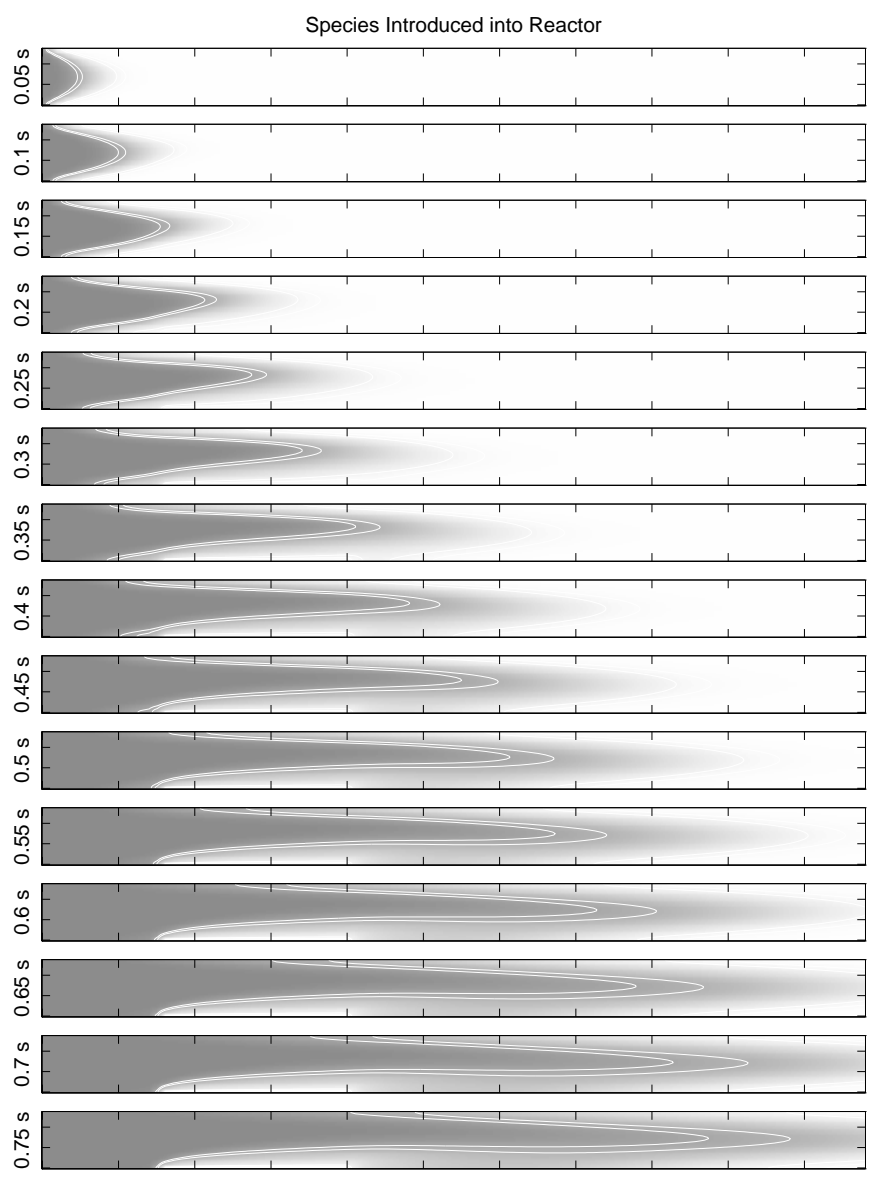

Figure 6: TMGa profile due to the continuous input of reactant at the inlet

(i) Obtain the snapshots. We first allow the chemical mixture of TMGa and $\mathrm{N}_{2}$ to enter the reactor at the inlet for 0.5 second. After 0.5 second, we shut off TMGa and allow only carrier gas, $\mathrm{N}_{2}$, to flow in for one second, during which we solve the species equation at 200 time steps (then snapshots) $\left\{c_{1}(\overrightarrow{\mathbf{x}}), c_{2}(\overrightarrow{\mathbf{x}}), \ldots, c_{200}(\overrightarrow{\mathbf{x}})\right\}$ at an increment of 0.005 second for $\overrightarrow{\mathbf{x}} \in \Omega$ (here, $\Omega$ denotes the two-dimensional rectangular domain as depicted in Figure 2). These snapshots are pointwise discrete data of species over $\Omega$, which have been computed using FIDAP. Some sample snapshots of species are displayed in Figure 9 . We note that at time $t=0.8$ second (see snapshot \# 160) most of the TMGa has been carried out of the reactor. 

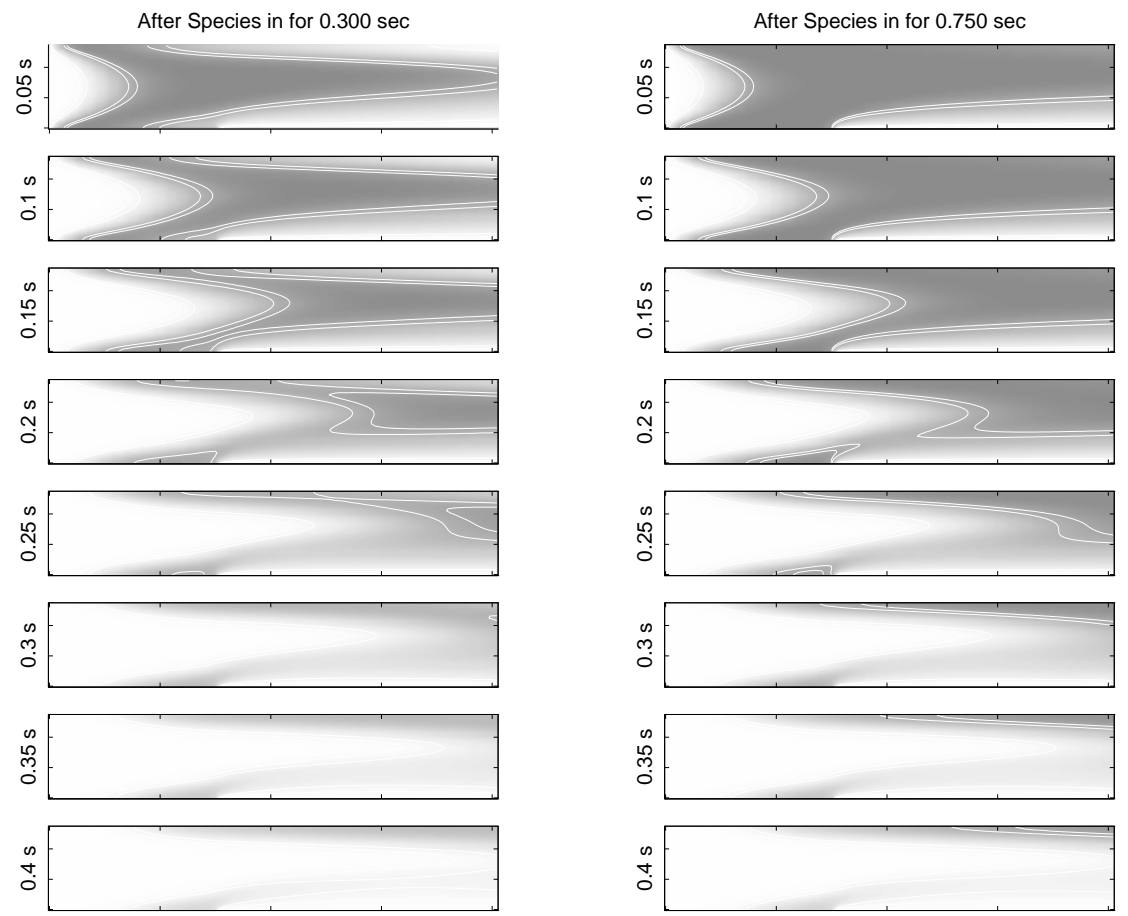

Figure 7: TMGa profiles corresponding to $\mathrm{T}_{\mathrm{in}}$ equals to .3 second (left) and 0.75 second (right)

(ii) Compute the covariant matrix $\mathbf{C}$. The matrix elements of $\mathbf{C}$ are given by

$$
\mathbf{C}_{i k}=\frac{1}{200} \int_{\Omega} c_{i}(\overrightarrow{\mathbf{x}}) c_{k}(\overrightarrow{\mathbf{x}}) d x,
$$

for $i, k=1,2, \ldots, 200$.

(iii) Solve the eigenvalue problem $\mathbf{C V}=\lambda \mathbf{V}$. We recall that since $\mathbf{C}$ is a nonnegative, Hermitian matrix, it has a complete set of orthogonal eigenvectors

$$
\mathbf{V}^{1}=\left[\begin{array}{c}
a_{1}^{1} \\
a_{2}^{1} \\
\vdots \\
a_{200}^{1}
\end{array}\right], \mathbf{V}^{2}=\left[\begin{array}{c}
a_{1}^{2} \\
a_{2}^{2} \\
\vdots \\
a_{200}^{2}
\end{array}\right], \ldots, \mathbf{V}^{200}=\left[\begin{array}{c}
a_{1}^{200} \\
a_{2}^{200} \\
\vdots \\
a_{200}^{200}
\end{array}\right]
$$

with the corresponding eigenvalues arranged in ascending order as $\lambda_{1} \geq$ $\lambda_{2} \geq \cdots \geq \lambda_{200} \geq 0$. 


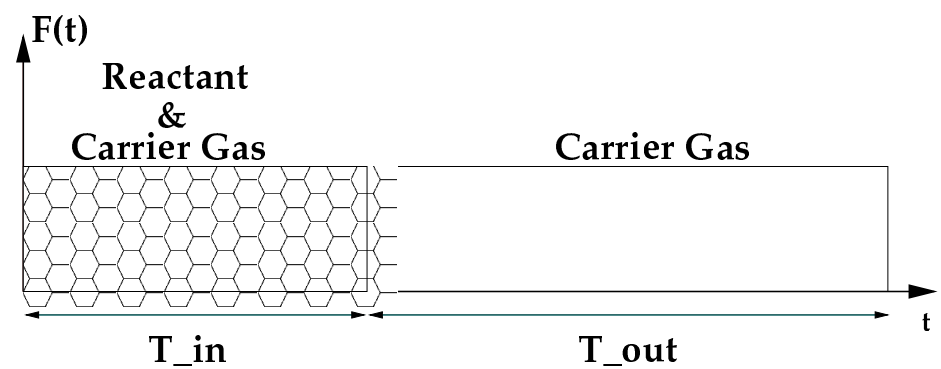

Figure 8: Schematic diagram for the boundary condition of TMGa at the inlet

(iv) Compute the POD basis vectors. The POD basis elements $\Phi_{i}(\overrightarrow{\mathbf{x}})$ such that $X^{\mathrm{POD}}=\operatorname{span}\left\{\Phi_{1}(\overrightarrow{\mathbf{x}}), \Phi_{2}(\overrightarrow{\mathbf{x}}), \ldots, \Phi_{200}(\overrightarrow{\mathbf{x}})\right\}$ are defined as

$$
\Phi_{k}=\sum_{i=1}^{200} a_{i}^{k} c_{i},
$$

where $1 \leq k \leq 200$ and $a_{i}^{k}$ are the elements of the eigenvector $\mathbf{V}^{k}$ corresponding to the eigenvalue $\lambda_{k}$.

\subsection{Reconstruction of Solutions Using POD Basis Vectors}

We next consider the species equation

$$
\frac{\partial c}{\partial t}+\overrightarrow{\mathbf{u}} \cdot \nabla c=\frac{1}{\rho} \nabla \cdot\left(\rho D_{T} \nabla c\right), \quad c(\overrightarrow{\mathbf{x}}, 0)=g(\overrightarrow{\mathbf{x}}),
$$

where $\overrightarrow{\mathbf{u}}$, and the temperature dependent parameters $\rho$, and $D_{T}$ are obtained from the steady state solutions of the coupled system (2.1)-(2.3) and (2.5) with boundary condition as described in Figure 4 . In this section, we will consider the problem of approximating the infinite-dimensional equation (4.2) by a sequence of finite-dimensional problems using a combination of Galerkin approximations and POD basis elements. We first formulate the species equation (4.2) in a variational or weak form; that is, we seek a solution $t \rightarrow c(t)$ on $0 \leq t \leq T$, with $c(t) \in H^{1}(\Omega)$ satisfying

$$
\left(c_{t}, \psi\right)+(\overrightarrow{\mathbf{u}} \cdot \nabla c, \psi)=\left(\frac{D_{T}}{\rho} \nabla c \cdot \nabla \rho, \psi\right)-\left(D_{T} \nabla c, \nabla \psi\right)+\int_{\partial \Omega} D_{T} \psi \nabla c \cdot \overrightarrow{\mathbf{n}} d s
$$

for all $\psi \in H^{1}(\Omega)$, along with initial condition

$$
c(0)=g .
$$

Here, $\overrightarrow{\mathbf{n}}$ denotes the unit outward normal vector to the boundary $\partial \Omega$ of the domain $\Omega$. 

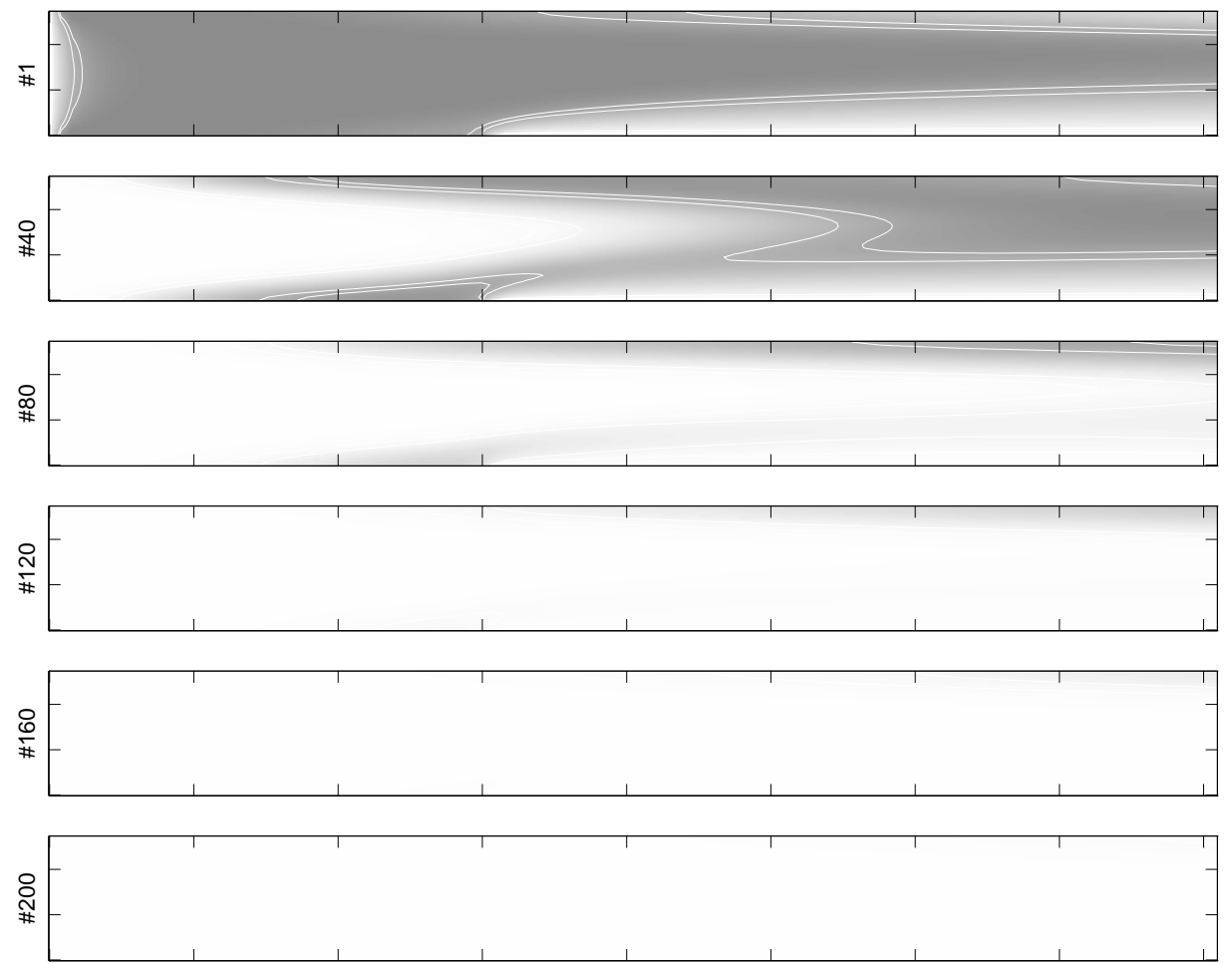

Figure 9: Snapshots \#1, 40, 80, 120, 160 and 200

As we have discussed in the previous section, fewer than 200 POD basis vectors are used to approximate the species solution thereby achieving a reduced order model. That is, the energy contained in the first $M$ POD modes, $M \ll$ 200 , represents most of the energy in the system, if we choose $M$ so that

$$
\sum_{i=1}^{M} \lambda_{i} \cong \sum_{i=1}^{200} \lambda_{i} .
$$

In our case, we found that the first 10 POD basis functions, displayed in Figure 10 , capture over $99.9 \%$ of the characteristics of the 200 observations. Here, for $i=1, \ldots, 10$, POD \# $\mathrm{i}$ refers to the graph of the function $\Phi_{i}(\overrightarrow{\mathbf{x}})$ for $\overrightarrow{\mathbf{x}} \in \Omega$. More precisely,

$$
\frac{\sum_{i=1}^{10} \lambda_{i}}{\sum_{i=1}^{200} \lambda_{i}}=.999 .
$$

Therefore, it is reasonable to approximate the species solution by using only 
the first 10 POD basis vectors, wherein we obtain a solution of the form

$$
c_{\mathrm{POD}}(\overrightarrow{\mathbf{x}}, t)=\sum_{i=1}^{10} \alpha_{i}(t) \Phi_{i}(\overrightarrow{\mathbf{x}}) .
$$
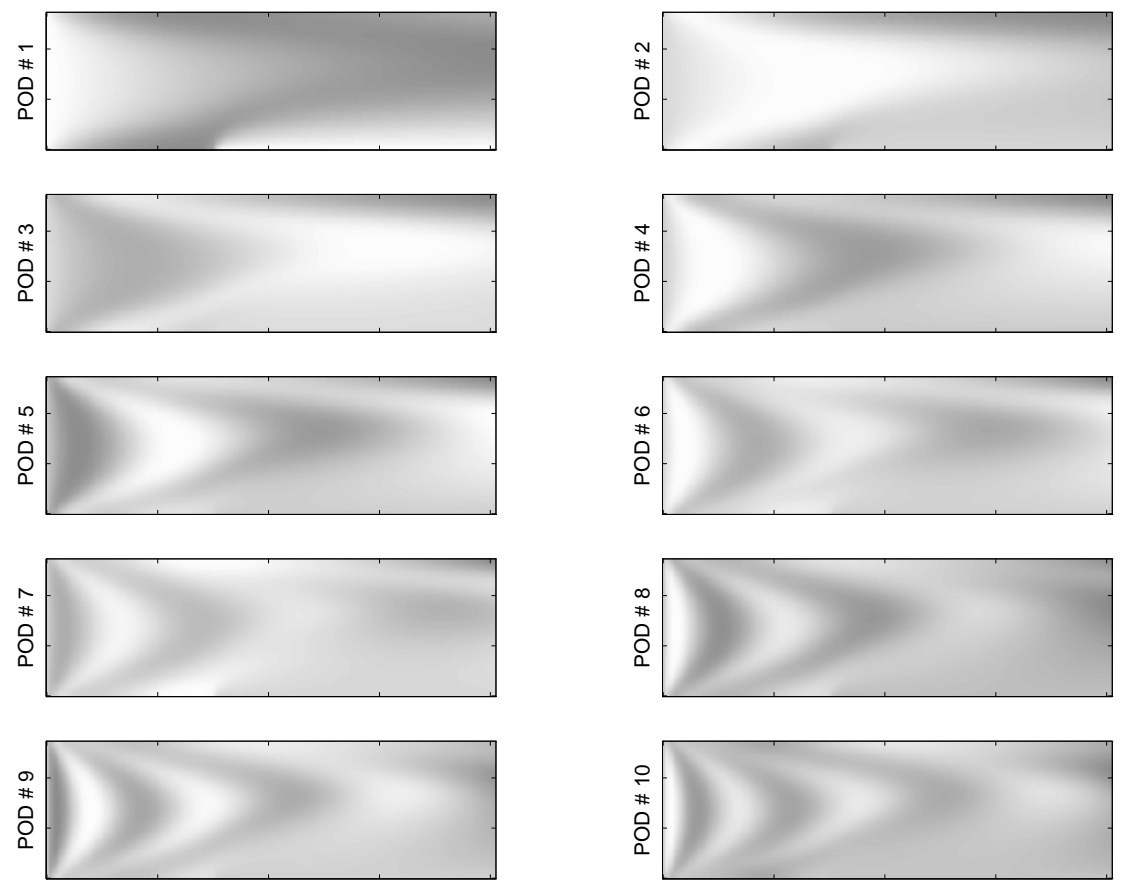

Figure 10: The first 10 POD elements

Using the form of $c(\overrightarrow{\mathbf{x}}, t)$ in (4.4) and the orthonormality of the $\left\{\Phi_{i}\right\}$ 's, we apply a Galerkin procedure to equation (4.2) to obtain a system of 10 ordinary differential equations for the coefficients $\alpha_{j}$

$$
\begin{aligned}
& \frac{d \alpha_{j}}{d t}=\mathbf{N}_{j}\left(\alpha_{1}, \alpha_{2}, \ldots, \alpha_{10}\right) \\
& \alpha_{j}(0)=\left(c\left(\overrightarrow{\mathbf{x}}, T_{\mathrm{in}}\right), \Phi_{j}\right),
\end{aligned}
$$

for $1 \leq j \leq 10$, where

$$
\begin{aligned}
\mathbf{N}_{j} & =\sum_{i=1}^{10}\left[\left(\frac{1}{\rho} \nabla \cdot\left(\rho D_{\mathrm{T}} \nabla \Phi_{i}\right), \Phi_{j}\right)-\sum_{i=1}^{10}\left(\overrightarrow{\mathbf{u}} \cdot \nabla \Phi_{i}, \Phi_{j}\right)\right] \alpha_{i}(t) \\
& =-\sum_{i=1}^{10}\left[\left(D_{\mathrm{T}} \nabla \Phi_{i}, \nabla \Phi_{j}\right)+\left(\frac{D_{\mathrm{T}}}{\rho} \nabla \Phi_{i} \cdot \nabla \rho, \Phi_{j}\right)-\left(\overrightarrow{\mathbf{u}} \cdot \nabla \Phi_{i}, \Phi_{j}\right)\right] \alpha_{i}(t) .
\end{aligned}
$$


The first of these equalities results from using (4.4) in a strong form of (4.2), multiplying by elements and integrating, while the equivalent second equality results from using (4.4) directly in the weak form (4.3) in the usual Galerkin procedure. The solutions to the initial value problem (4.5) yield the coefficients of the POD basis function approximation (4.4).

\subsection{Simulation Results}

In this section, we examine the accuracy and efficiency of the low-dimensional dynamical model (4.5) obtained from the Galerkin procedure employing POD basis functions by comparing its solution with the solution obtained from the fluid dynamics package FIDAP. More specifically, in FIDAP the domain $\Omega$ is discretized using 2400 (9-nodal quadratic) quadrilateral elements. Consequently, by using FIDAP, a system of 14,801 ordinary differential equations has to be solved for the coefficients of the basis function approximation. We point out again that in our formulation using a Galerkin procedure with POD basis functions, the resulting approximation is a system of 10 ordinary differential equations for the coefficients of the POD basis function approximation (4.4).

Figure 11 compares the reduced solution using $10 \mathrm{POD}$ basis functions to the full solution obtained from FIDAP employing 2400 (9-nodal quadratic) quadrilateral finite elements. Specifically, both solutions are obtained with boundary condition

$$
\left.c(\overrightarrow{\mathbf{x}}, t)\right|_{\text {inlet }}= \begin{cases}F(t)=1 & 0<t \leq \mathrm{T}_{\mathrm{in}}, \\ 0 & \mathrm{~T}_{\mathrm{in}}<t,\end{cases}
$$

where $T_{\text {in }}=0.5$ second. Qualitatively, the reduced POD basis results agrees favorably with the full FIDAP calculations. Quantitatively, the upper plot in Figure 12 graphs the $L_{2}$ norms of FIDAP solutions, $\left\|c_{\mathrm{FIDAP}}(\cdot, t)\right\|_{L_{2}(\Omega)}$, and the $L_{2}$ absolute errors between POD solutions and FIDAP solutions $\| c_{\mathrm{POD}}(\cdot, t)-$ $c_{\text {FIDAP }}(\cdot, t) \|_{L_{2}(\Omega)}$. The bottom plot in Figure 12 graphs the $L_{2}$ norms of FIDAP fluxes, $\left\|\mathcal{F}_{\mathrm{FIDAP}}(\cdot, t)\right\|_{L_{2}(\Omega)}$, and the $L_{2}$ absolute errors between POD fluxes and FIDAP fluxes $\left\|\mathcal{F}_{\mathrm{POD}}(\cdot, t)-\mathcal{F}_{\mathrm{FIDAP}}(\cdot, t)\right\|_{L_{2}\left(\Omega_{s}\right)}$ Here, the flux of the species above the susceptor $\left(\Omega_{s}\right)$ that dictates the rate at which species arrives at the surface is defined by

$$
\mathcal{F}(\overrightarrow{\mathbf{x}}, t):=\left.\left(D_{\mathrm{T}} \nabla c(\overrightarrow{\mathbf{x}}, t)+\rho c(\overrightarrow{\mathbf{x}}, t) \overrightarrow{\mathbf{u}}\right) \cdot \overrightarrow{\mathbf{n}}\right|_{\overrightarrow{\mathbf{x}} \in \Omega_{s}} .
$$

The flux of species above the susceptor is used in the next section to define the cost functional in an optimal control problem. Therefore, it is important that this quantity can be computed accurately. For a comparison, Figure 13 depicts the fluxes at the susceptor computed using POD basis functions and FIDAP software package. Both results agree remarkably well. 

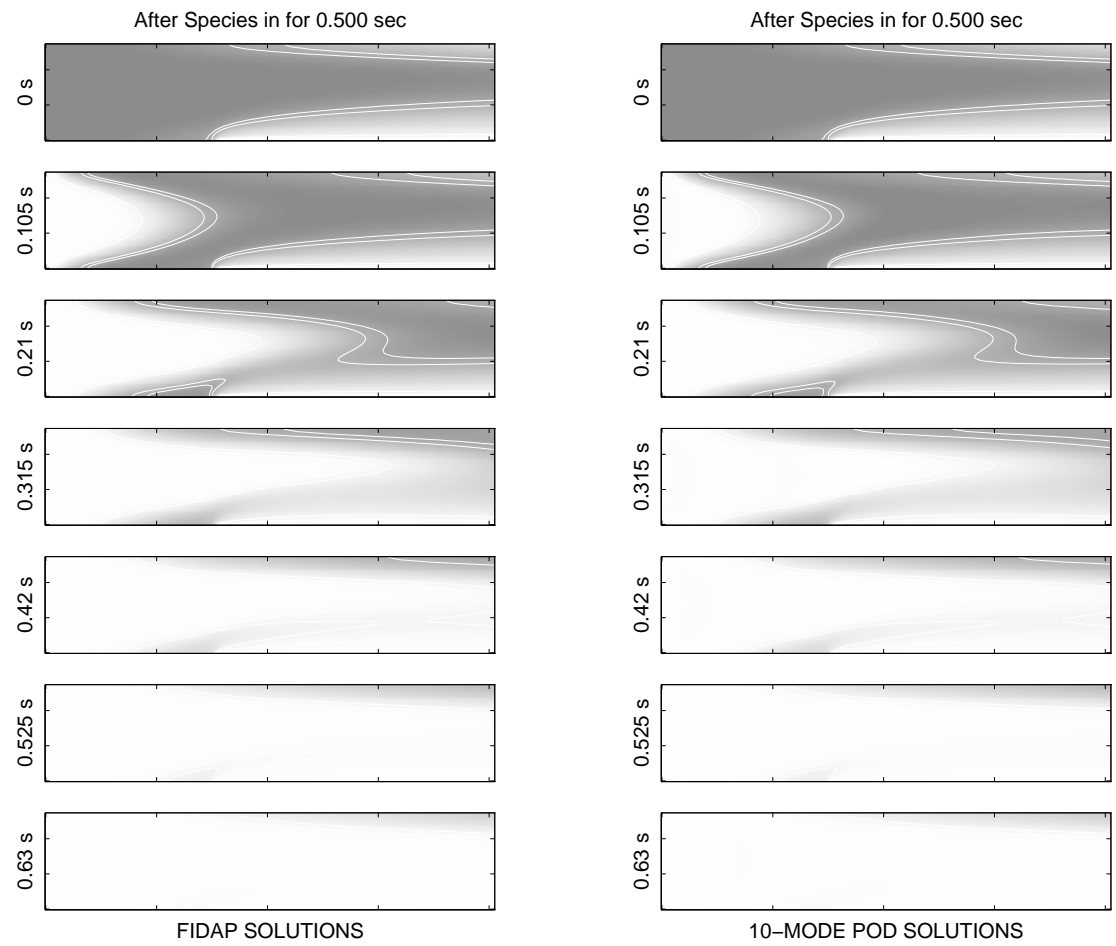

Figure 11: FIDAP Solution (left) and POD solution (right) at different times 

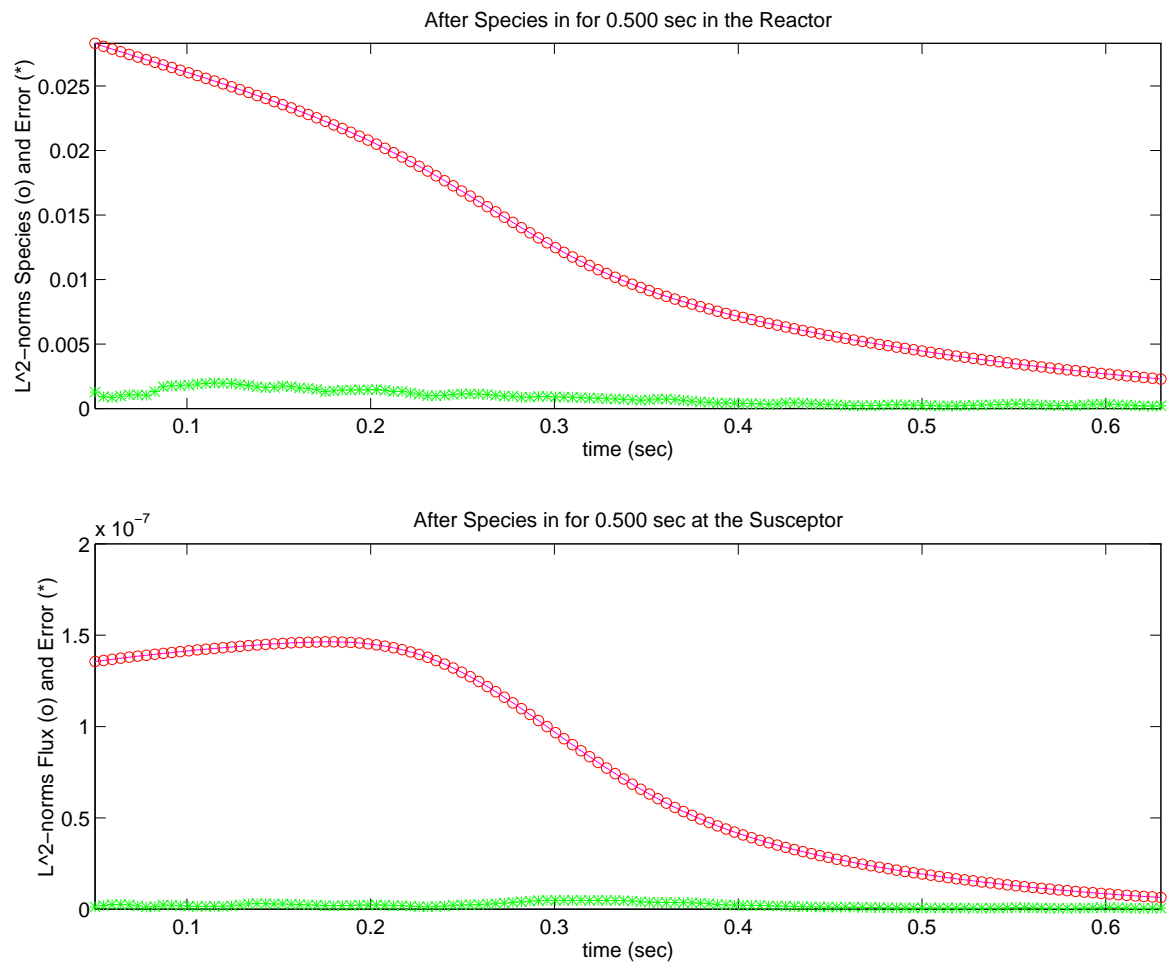

Figure 12: Top: the $L_{2}$-norm of FIDAP solution (o), $\left\|c_{\text {FIDAP }}(\cdot, t)\right\|$, and the $L_{2}$-norm error $\left(^{*}\right),\left\|c_{\mathrm{POD}}(\cdot, t)-c_{\mathrm{FIDAP}}(\cdot, t)\right\|$. Bottom: the $L_{2}$-norm of FIDAP flux (o), $\left\|\mathcal{F}_{\text {FIDAP }}(\cdot, t)\right\|$, and the $L_{2}$-norm of the flux error $\left({ }^{*}\right), \| \mathcal{F}_{\mathrm{POD}}(\cdot, t)-$ $\mathcal{F}_{\text {FIDAP }}(\cdot, t) \|$ 

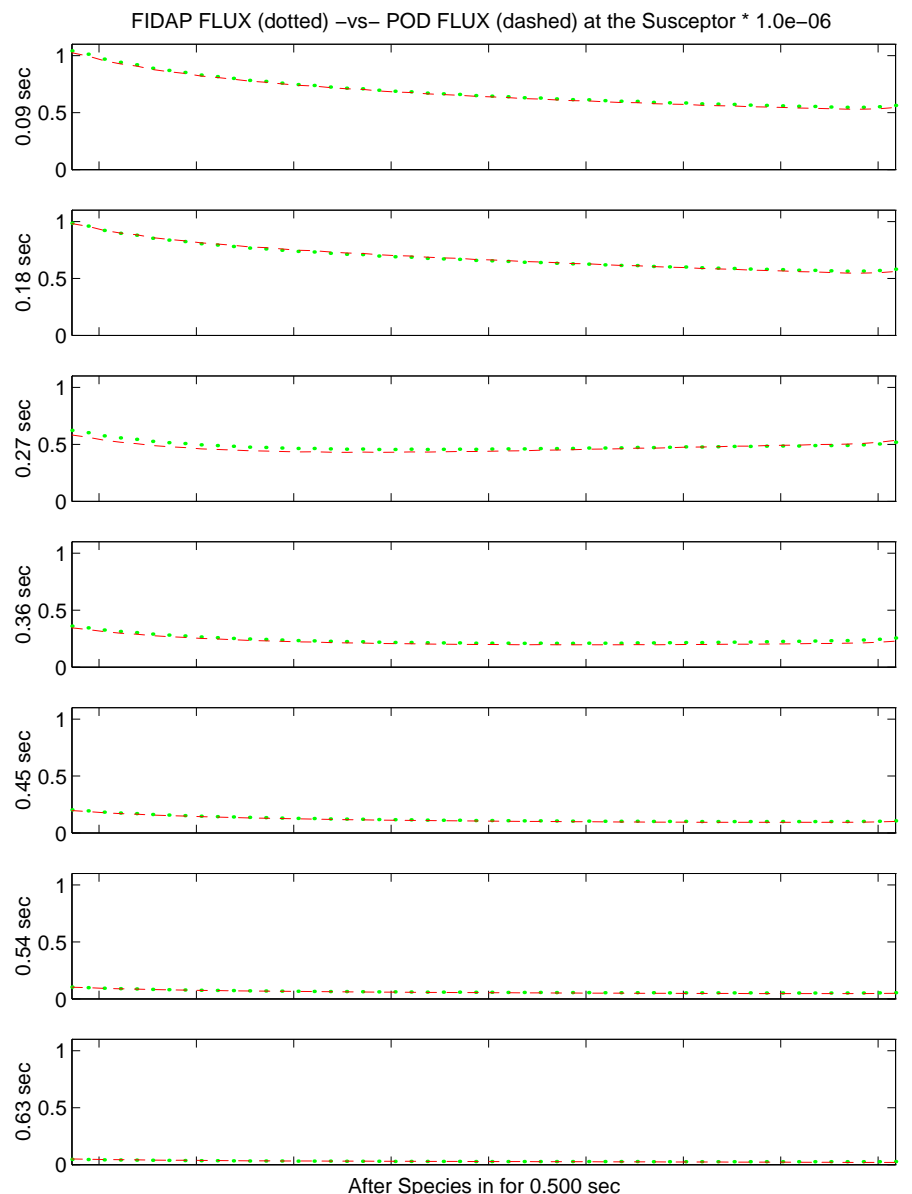

Figure 13: Fluxes of species at the susceptor using FIDAP (.) and POD basis functions (-) at different times

We recall that POD basis functions were computed from the snapshots corresponding to the boundary condition (4.3) with $T_{\mathrm{in}}=0.5$ second. To be useful in developing both open loop and feedback control strategies, we would like for these same reduced number of POD elements to yield good approximations under other flow input conditions. That is, we would like to demonstrate that using exactly these 10 POD modes, we are able to construct POD solutions for different durations of species introduced into the reactor. Particularly, we solved the same system (4.5) with initial conditions $\alpha_{j}(0)=\left(c\left(\overrightarrow{\mathbf{x}}, T_{\text {in }}\right), \Phi\right)$ for $T_{\text {in }}=0.3$ second and $T_{\text {in }}=0.75$ second. Figure 14 depicts the $L_{2}$-norms of the solution errors and $L_{2}$-norms of the flux errors above the susceptor using POD basis functions and FIDAP for $T_{\text {in }}=0.30$ second and $T_{\text {in }}=0.75$ second. 

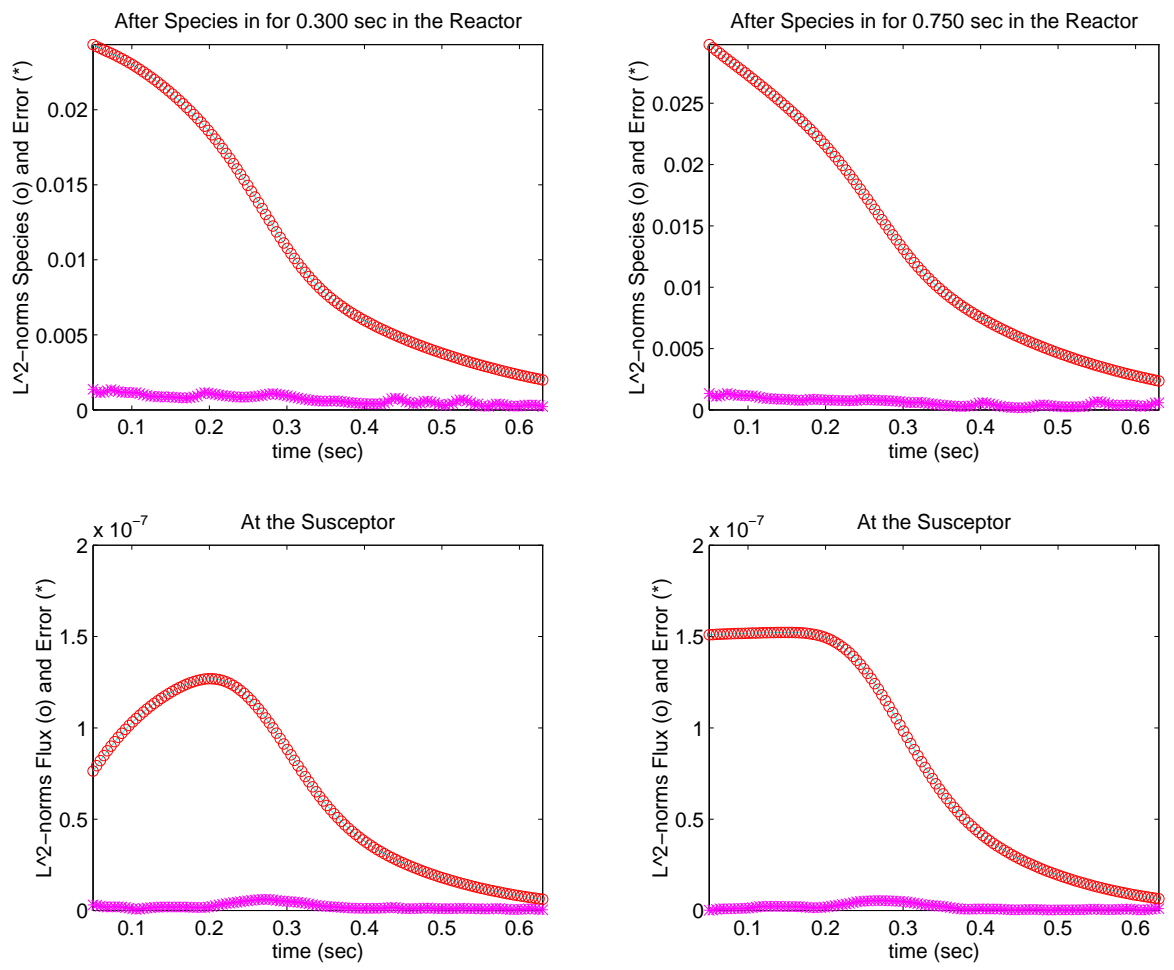

Figure 14: (left columm, $T_{\mathrm{in}}=0.3$ second, right columm, $T_{\mathrm{in}}=0.75$ second): Top, the $L_{2}$-norm of FIDAP solution (o), $\left\|c_{\text {FIDAP }}(\cdot, t)\right\|$, and the solution error $\left(^{*}\right),\left\|c_{\mathrm{POD}}(\cdot, t)-c_{\mathrm{FIDAP}}(\cdot, t)\right\|$; Bottom, the $L_{2}$-norm of FIDAP flux $(\mathrm{o})$, $\left\|\mathcal{F}_{\text {FIDAP }}(\cdot, t)\right\|$, and the $L_{2}$-norm of the flux error $\left(^{*}\right),\left\|\mathcal{F}_{\mathrm{POD}}(\cdot, t)-\mathcal{F}_{\operatorname{FIDAP}}(\cdot, t)\right\|$

\section{An Optimal Control Problem}

In this section we demonstrate the use of reduced POD models in an open loop optimal control problem. While this example involves only one control variable, it does illustrate effectively the potential of POD methods in control problems.

In the case of GaN heteroepitaxy film growth employing pulsed trimethylgallium (TMGa) and ammonia $\left(\mathrm{NH}_{3}\right)$ as source vapors, depending on the delay between the TMGa and $\mathrm{NH}_{3}$ source vapor pulses, carry-over of TMGa fragments from one precursor pulse cycle to the next may occur. This, in turn, establishes a surface reaction layer (SRL), consisting of mixture of reactants and products of the chemical reactions that drive the epitaxial growth process. The thickness and composition of the SRL depends on the relative heights and widths, i.e. $T_{\mathrm{in}}$, of the employed $\mathrm{TMGa}$ and $\mathrm{NH}_{3}$ source vapor pulses and their repetition rate. More specifically, if $T_{\text {in }}$ is small, then the flux above the susceptor is uniform. Yet this will take a long time to grow a film which makes 
it inviable in industrial applications. On the other hand, by allowing massive chemical input at the inlet (i.e., $T_{\text {in }}$ is large) we will speed up the growing process; but the flux above the susceptor may not be uniform. Therefore it is desirable to perform an optimization procedure to determine the most desirable $T_{\text {in }}$ to achieve both film uniformity and fastest possible growth rate. Mathematically, we would like to find the optimal $T_{\text {in }}$ so that the flux fluctuation, $\frac{\partial}{\partial x} \mathcal{F}$, is small while at the same time we maximize the flux, $\mathcal{F}$, to the substrate. Consequently, one way to formulate a quantitative representation of these conflicting desires is to seek $T_{\text {in }}^{*}$ so that the cost functional

$$
\text { Cost }=I+\frac{\alpha}{J}, \quad \alpha=\frac{1}{5.0 \times 10^{12}},
$$

is minimized, where

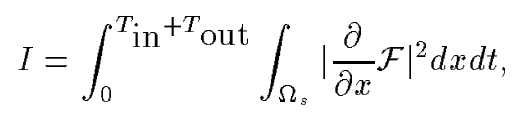

and

$$
J=\int_{0}^{T_{\text {in }}{ }^{+T} \text { out }} \int_{\Omega_{s}}|\mathcal{F}|^{2} d x d t
$$

Here, $T_{\text {out }}$ denotes the time delay between the source vapor pulses and, based on experimental results, we take $T_{\text {out }} \approx 0.600$ second. The above cost functional (5.1) is minimized subject to the system (2.2)-(2.5) along with their boundary conditions. The constant $\alpha$ in (5.1) is scaled to make $I$ and $\frac{1}{J}$ proportioned. It also bears the trade-off value which represents on one's desires to balance film uniformity and faster growth rate.

Given a value of $T_{\text {in }}$ the system of equations (2.2)-(2.5) is solved for the species solution which is then substituted into the formula (4.3) for the flux, $\mathcal{F}$. Hence, the above optimization problem is an unconstrained minimization problem involving one parameter, $T_{\text {in }}$. We carried out computations for such a control example. For these, we chose the DUVMGS subroutine in the Fortran IMSL library [1989] which is designed to minimize a nonsmooth function with double-precision accuracy. The subroutine DUVMGS uses the so-called golden section method to search for its minimal point. Other choices of minimization techniques are possible. We found that with the value of $\alpha$ given in (5.1), the optimal width of the pulses, $T_{\text {in }}^{*}$, is 0.395 second. The time-dependent fluxes above the susceptor for the optimal $T_{\text {in }}^{*}$ are compared in Figure 15 with nonoptimal fluxes computed using $T_{\mathrm{in}}=0.300$ second and $T_{\mathrm{in}}=0.650$ second. We note that $T_{\text {in }}$ must be at least .300 second which is the fastest on-off switch in the flow control panel. The flux above the susceptor for $T_{\mathrm{in}}=0.300$ second is uniform, i.e., the curve is flat, however, the growth rate is small. On the other hand, for $T_{\text {in }}=0.650$ second, the growth rate speeds up, but non-uniformity of film growth results. The optimal solution lies between the above two nonoptimal curves. This solution which corresponds to the optimal duration of the source vapor pulses achieves both film uniformity and fastest possible growth rate. 

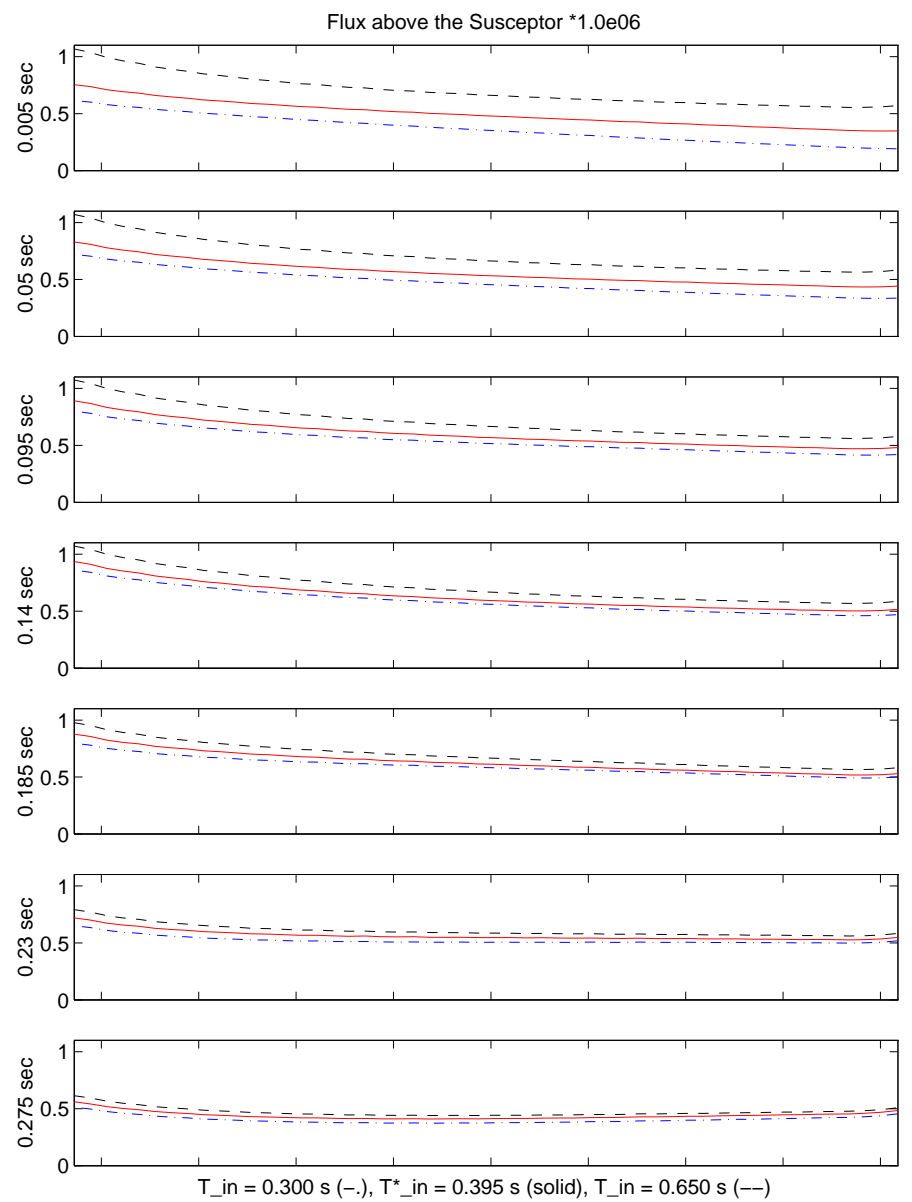

Figure 15: Comparing the optimal flux solution with two non-optimal flux solutions

\section{Acknowledgment.}

The authors is grateful to Dr. Grace M. Kepler for helping us to set-up FIDAP7.6 for obtaining the snapshots and to Drs. K.J. Bachman, H.T. Banks, C. Hoepfner and J.S. Scroggs for many helpful discussions.

\section{References}

[1] N. Aubry, P. Holmes, J.L. Lumley, and E. Stone, "The dynamics of coherent structures in the wall region of a turbulent boundary layer," Journal of Fluid Mechanics, 192 (1988) p. 115-173. 
[2] N. Aubry, W.Y. Lian, and E.S. Titi, "Preserving symmetries in the proper orthogonal decomposition," SIAM J. Sci. Comput., 14, No. 2 (1993) p. 483-505.

[3] K.J. Bachmann, N. Sukidi, C. Hopfner, C. Harris, N. Dietz, H.T. Tran, S. Beeler, K. Ito, and H.T. Banks "Real-time monitoring of steady-state pulsed chemical beam epitaxy by p-polarized reflectance," J. of Crystal Growth, 183 (1998) p. 323-337.

[4] K.S. Ball, L. Sirovich, and L.R. Keefe "Dynamical eigenfunction decomposition of turbulent channel flow," International Journal for Numerical Methods in Fluids, 12 (1991) p. 585-604.

[5] H.T. Banks, K. Ito, J.S. Scroggs, H.T. Tran, N. Dietz, and K.J. Bachmann, "Modeling and control of advanced chemical vapor deposition processes," in "Mathematics of Microstructure Evolution," (eds.: L.Q. Chen, et al), SIAM/TMS Publications, (1996) p. 327-344.

[6] G. Berkooz, "Observations on the proper orthogonal decomposition," in "Studies in Turbulence," (eds.: T.B. Gatski, S. Sarkar, and C.G. Speziale), Springer-Verlag, New York (1992) p. 229-247.

[7] G. Berkooz, P. Holmes, and J.L. Lumley, "The Proper orthogonal decomposition in the analysis of turbulent flows," Annual Review of Fluids Mechanics, 25 (1993) N5:539-575.

[8] G. Berkooz, P. Holmes J.L. Lumley, and J.C. Mattingly, "Low-dimensional models of coherent structures in turbulence," Physics Reports-Review Section of Physics Letters, 287 (1997) N4:338-384.

[9] D.H. Chambers, R.J. Adrian, P. Moin, D.S. Stewart, and H.J. Sung, "Karhunen-Loève expansion of Burgers' model of turbulence," Phys. Fluids, 31 (1988) p. 2573-2582.

[10] M.E. Coltrin, R.J. Kee, and J.A. Miller, "A mathematical model of Silicon chemical vapor deposition," J. Electrochem. Soc., 133 (1986) p. 1206-1213.

[11] D.I. Fotiadis, "Two- and three-dimensional finite element simulations of reacting flows in chemical vapor deposition of compound semiconductors," PhD thesis,, Univ. Minn., Minneapolis, (1990).

[12] E. Fujii, H. Nakamura, K. Haruna, and Y. Koga, "A quantitative calculation of the growth rate of epitaxial silicon from $\mathrm{SiCl}_{4}$ in a barrel reactor," J. Electrochem. Soc., 119 (1972) p. 1106-1113.

[13] S.A. Gokoglu, M. Kuczmarski, P. Tsui, and A. Chait, "Convection and chemistry effects in CVD - A 3-D analysis for silicon deposition," Journal de Physique, 50 (1989) N5-17.

[14] M. Graham and I.G. Kevrekidis, "Alternative approaches to the KarhunenLoève decomposition for model reduction and data analysis," Computers Chem. Engineering, 20 (1996) N5:495-506. 
[15] R. Hilai and J. Rubinstein, "Recognition of rotated images by invariant Karhunen-Loève expansion," Journal of the Optical Society of America A-Optics Image Science and Vision, 11 (1994) N5:1610-1618.

[16] K.Ito, J.S. Scroggs, and H.T. Tran, "Mathematical Issues in Optimal Design of a Vapor Transport Reactor", in IMA Volumes in Mathematics and Its Applications, 68, Flow Control (Max D. Gunzburger, ed.), (1994) p. $197-218$.

[17] K.Ito, J.S. Scroggs, and H.T. Tran, "Optimal Control of Thermally Coupled Navier-Stokes Equation", in Optimal Design and Control, Max D. Gunzburger et al., eds.), (1995) p. 199-214.

[18] K.F. Jensen, "Chemical vapor deposition,", in Microelectronics Processing: Chemical Engineering Aspects, D.W. Hess and K.F. Jensen, eds., (1989) p. 199-263.

[19] K.F. Jensen, E.O. Einset, and D.I. Fotiadis, "Flow phenomena in chemical vapor deposition of thin films," Annu. Rev. Fluid Mech., 23 (1991) p. 197232 .

[20] R.P. Kanwal, "Linear Integral Equations: Theory \& Technique", Birkhäuser, Boston (1997).

[21] K. Karhunen, "Zur spektral theorie stochasticher prozesse," Ann. Acad. Sci. Fennicae, Ser. A1 Math Phys., 37 (1946).

[22] M. Kirby and L. Sirovich, "Application of the Karhunen-Loève procedure for the characterization of human faces," IEEE Transactions on Pattern Analysis and Machine Intelligence, 12 (1990) N1:103-108.

[23] M. Kirby, J.P. Boris, and L. Sirovich, "A proper orthogonal decomposition of a simulated supersonic shear layer," International Journal for Numerical Methods in Fluids, 10 (1990) p. 411-428.

[24] M. Loève, "Functions aleatoire de second ordre", "Compte Rend. Acad. Sci. (Paris)", (1945) p. 220.

[25] J.L. Lumley, "The structure of inhomogeneous turbulent flows," in "Atmospheric turbulence and radio wave propagation", A.M. Yaglom and V.I. Tatarski, eds.: Moscow: Nauka (1967) p. 166-178.

[26] J.L. Lumley, “Stochastic Tools in Turbulence," Academic Press, New York (1970).

[27] H.V. Ly and H.T. Tran, "Applications of proper orthogonal decomposition in simulations and optimal control of the Rayleigh-Bénard Convection," 1998 , to be submitted.

[28] H.K. Moffat and K.F. Jensen, "Complex flow phenomena in MOCVD reactors," Journal of Crystal Growth, 77 (1986) p. 108-119. 
[29] H.K. Moffat and K.F. Jensen, "Three-dimensional flow effects in Silicon CVD in horizontal reactors," J. Electrochem. Soc., 135 (1988) p. 459-471.

[30] P. Moin and R.D. Moser, "Characteristic-eddy decomposition of turbulence in a channel," Journal Fluids Mechanics, 200 (1989) p. 417-509.

[31] A.M. Obukhov, "Statistical description of continuous fields", T. Geophys. Int. Akad. Nauk. USSR, 24 (1954), p. 3-42.

[32] S. Ostrach, "Low-gravity fluid flows," Ann. Rev. Fluid Mech., 14 (1982) p. $313-345$.

[33] K. Oswatitsch, “Gas Dynamics,” Academic Press, New York (1956).

[34] J. Ouazzani, Kuan-Cheng Chiu, and F. Rosenberger, "On the 2D modeling of horizontal CVD reactors and its limitations," Journal of Crystal Growth, 91 (1988) p. 497-508.

[35] J. Ouazzani and F. Rosenberger, "Three-dimensional modeling of horizontal chemical vapor deposition I. MOCVD at atmospheric pressure," Journal of Crystal Growth, 100 (1990) p. 545-576.

[36] R. Pollard and J. Newman, "Silicon deposition on a rotating disk," J. Electrochem. Soc.," 127 (1980) p. 744-752.

[37] V.S. Pougachev, "General theory of the correlations of random functions," Izv. Akad. Naul. USSR, Ser. Mat., 17 (1953) p. 1401-2.

[38] M. Rajaee, S.K.F. Karlson, and L. Sirovich, "Low-dimensional description of free-shear-flow coherent structures and their dynamical behavior," Journal of Fluid Mechanics, 258 (1994) p. 1-29.

[39] R.S. Reichert, F.F. Hatay, S.Biringer, and A. Husser, "Proper orthogonal decomposition applied to turbulent flows in a square duct," Phys. Fluids Mechanics, 6 (1994) N9:3086-3092.

[40] M.A. Saad, “Compressible Fluid Flow," Prentice-Hall, New Jersey (1985).

[41] L. Sirovich, "Chaotic dynamics of coherent structures," Physica D, 37 (1989) p. 126-145.

[42] L. Sirovich, "Analysis of turbulent flows by means of the empirical eigenfunctions," Fluid Dynamics Research, 8 (1991) p. 85-100.

[43] J.S. Scroggs, H.T. Banks, K. Ito, S. Ravindran, H.T. Tran, K.J. Bachmann, H. Castleberry, and N. Dietz, "High pressure vapor transport of $Z n G e P_{2}$ : $I I$, three-dimensional simulation of gasdynamics under microgravity conditions," in "Proceedings of the 1995 TMS Annual Meeting,", Las Vegas, Nevada, (1995).

[44] T. Theodorsen, "Mechanism of Turbulence," in "Proc. 2nd Midwestern Conf. on Fluid Mechanics," Ohio State University, Columbus, OH (1952). 
[45] A.A. Townsend, "The Structure of Turbulent Shear Flow," University Press, Cambridge, (1956).

[46] G.W. Young, S.I Hariharan, and R. Carnahan, "Flow effects in a vertical CVD reactor," SIAM J. Appl. Math., 52, No. 6, (1992) p. 1509-1532. 